\title{
Piezoelectric Semiconducting Nanowires
}

\author{
Yonatan Calahorra, Canlin Ou, Chess Boughey and Sohini Kar-Narayan \\ Department of Materials Science and Metallurgy, University of Cambridge, Cambridge, \\ $U K$
}

\section{Introduction}

Semiconductor nanowires have been at the forefront of nanotechnology, bringing forth advances in electronics, optoelectronics and basic physics, to name a few. More recently, there has been increasing interest in the existence of piezoelectricity in some of these materials, whereby they are capable of inter-converting mechanical and electrical energy. Piezoelectricity has been found to manifest more strongly in nanowires of some semiconductor materials as compared to the bulk, which makes nanowires particularly attractive for applications in strain sensing and mechanical energy harvesting. At the same time, the geometry and unclamped nature of nanowires render them sensitive to small forces/deflections, and hence there has been an ongoing effort to incorporate them into so-called piezoelectric nanogenerators that are capable of harvesting energy from ambient vibrations to power autonomous devices, such as ubiquitous wireless sensor nodes. Furthermore, semiconducting piezoelectric materials have been driving the emerging fields of piezotronics and piezo-phototronics, where the development of a piezopotential in response to stress or strain can be used to tune the electronic and/or optical properties of the material by altering the band structure of the semiconductor. There are many potential sensing and energy-based applications that can arise from the development of this area of research, but only a few candidate semiconducting materials systems have been explored thus far for their piezotronic and photo-piezotronic properties. Further development of the field requires investigation and development of a wide range of piezoelectric semiconducting nanowires. However, the direct measurement of piezoelectric properties of these nanowires is challenging, and thus rarely reported. This is due to generic difficulties associated with measurements of piezoelectric properties of nanoscale objects using conventional scanning 
probe microscopy techniques, which present key characterization challenges in this field. Recent advances in nanoscale characterization of piezoelectric semiconducting nanowires, such as the development of non-destructive piezoresponse force microscopy (ND-PFM), are opening up new avenues into the detailed investigation of nanoscale piezoelectricity in these materials. This chapter provides an overview of piezoelectric semiconducting nanowires, with a focus on the nature of the effect itself, how it manifests in different materials, how it is measured at the nanoscale, and finally different types of piezoelectric energy-related devices that can be achieved using these nanowires. A greater understanding of the underlying physics that governs piezoelectricity in semiconducting nanowires will serve to underpin further developments of their applications in energy devices.

\section{Background}

\subsection{Piezoelectricity}

The piezoelectric effect results from relative displacement of ions or polar components within a piezoelectric material due to an applied stress, resulting in a change in net polarization. Piezoelectricity, first described by the Curie brothers [1], refers to the linear interrelation between the electrical and mechanical states of certain materials. Piezoelectricity is manifested in 20 out of the 21 non-centrosymmetric crystal classes, with the exception being the 432 cubic system. Centrosymmetric crystal classes do not exhibit piezoelectricity. Piezoelectricity is manifested as both a "direct" effect, where polarization is created in response to an applied stress, and a "converse" effect, where an applied electric field creates a strain within the material. Piezoelectricity is described mathematically with a set of complementary equations given below:

$$
\begin{aligned}
& D=\epsilon_{T} E+d T \\
& S=d^{t} E+s_{E} T
\end{aligned}
$$

where $T$ and $S$ represent stress and strain respectively, which are second rank tensors related to an applied force and the normal to the area over which it acts, $D$ and $E$ represent the electric displacement and electric field vectors, $\epsilon$ is the dielectric permittivity of the material, where subscript $T$ represents constant stress, $d$ is the piezoelectric tensor (third rank), and $d_{T}$ is the transpose of $d . s_{E}$ is the elastic compliance at constant electric field. 
These terms are second, third and fourth rank tensors respectively, and due to symmetry considerations Voigt notation is often used for convenience to describe the tensors. For the piezoelectric tensor this takes the form of a $3 \times 6$ matrix. There are four types of piezoelectric coefficients

$$
\begin{aligned}
d_{i j} & =\left(\frac{\partial D_{i}}{\partial T_{j}}\right)^{E} \\
e_{i j} & =\left(\frac{\partial D_{i}}{\partial S_{j}}\right)^{E} \\
g_{i j} & =-\left(\frac{\partial E_{i}}{\partial T_{j}}\right)^{D} \\
h_{i j} & =-\left(\frac{\partial E_{i}}{\partial S_{j}}\right)^{D}
\end{aligned}
$$

where the superscript indicates differentiation under constant or zero field. $d_{i j}$ and $e_{i j}$ are the most commonly used coefficients, often coined the piezoelectric strain $(d)$ and charge coefficients $(e) . i$ ranges from 1 to 3 and $j$ from 1 to 6 (Voigt), where 1,2,3 are direction axes and the 3 -axis typically denotes the polarization direction, while 4,5,6 denote shear deformation about 1,2,3 axes, correspondingly. $d_{i j}$ therefore describes polarization arising in the $i$ direction in response to stress along the $j$ direction, or alternatively strain in the $j$ direction in response to an electric field along the $i$ direction.

\subsection{Piezoelectric Semiconductors}

The most common semiconductors, with the exception of silicon and germanium, are piezoelectric; these include III-Vs and II-VIs. The wurtzite GaN family of materials, and $\mathrm{ZnO}$ are best known for their piezoelectric properties. Piezoelectricity in semiconductor materials can bring about both advantages and disadvantages. Unlike cubic structured GaAs/AlGaAs high electron mobility transistor (HEMT) technology which relies on the separation of dopants from the charge carriers [2], GaN/AlGaN HEMT technology is based on the piezoelectricity and spontaneous polarization discontinuities in III-N heterostrucutres, and does not rely on doping to achieve conductance (Figure 1) [3]. Interestingly, in the more established GaN LED lighting technology, the existence of piezoelectricity and spontaneous polarization is undesired, reducing emission efficiency [4]. This particular issue is directing research 
efforts towards "non-polar" III-N technology, and polarization-matched layers. For an exhaustive discussion on polarization in semiconductors, consult Ref. [5]
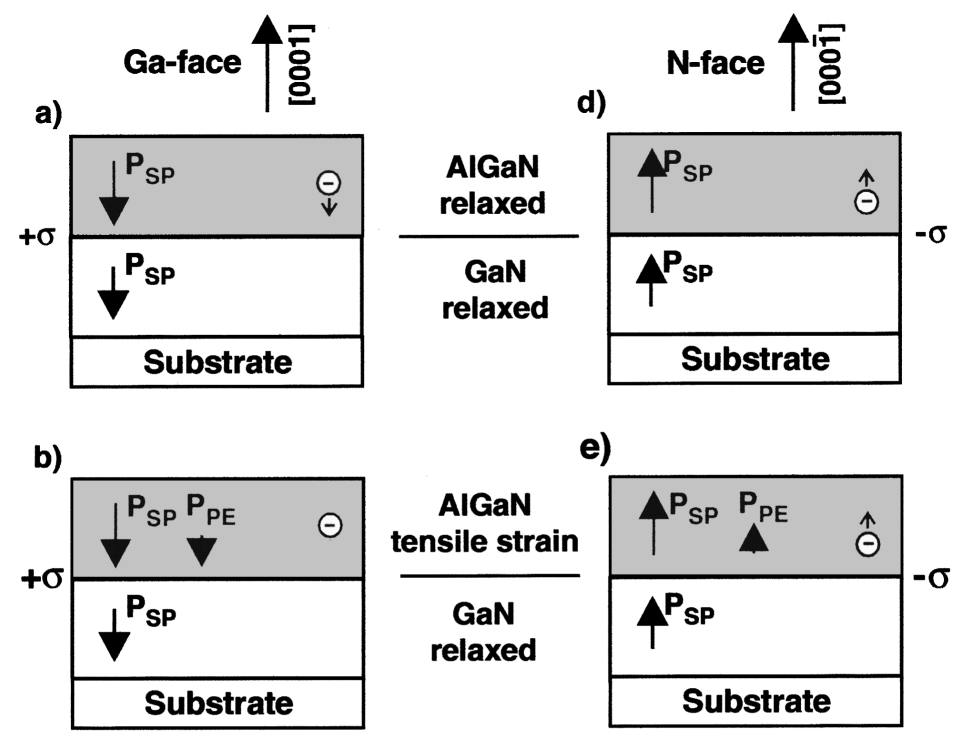

e)
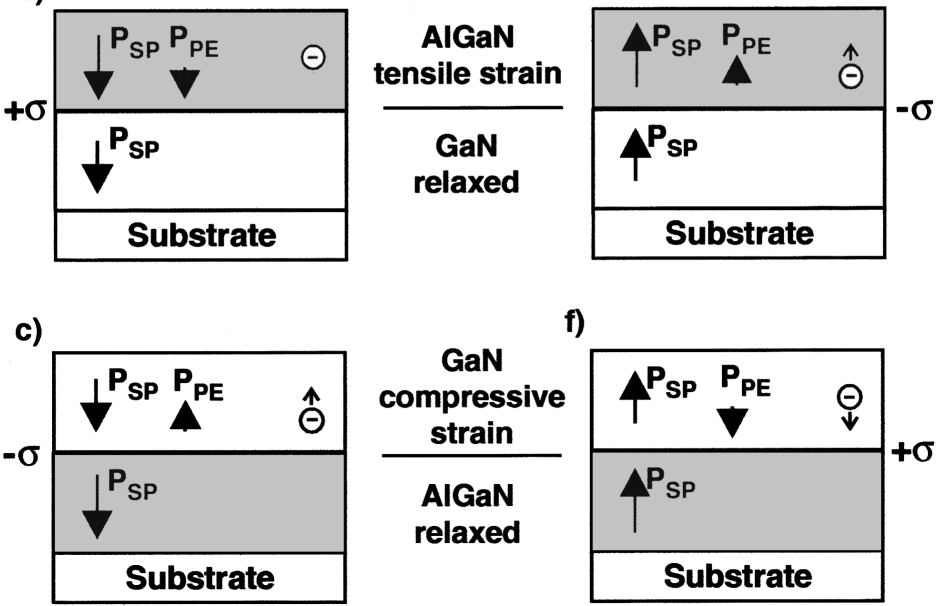

Figure 1: Schematic of the interplay between spontanous polarization, piezoelectricity and crystal orientation in the GaN/AlGaN heterostructure sytem, leading to the creation of a two-dimensional electron gas at the interface. Reproduced with permission from AIP [3].

Semiconductors include bound and free charges, and the relation between the charges and external and internal potentials is dominated by Poisson's 
equation and Fermi-Dirac distribution [6], taking the following form:

$$
\begin{aligned}
-\nabla^{2} \phi=\nabla \cdot D & =\rho \\
\rho & =q \cdot\left(N_{D}^{+}-N_{A}^{-}+p-n\right) \\
n & =\frac{2}{\sqrt{\pi}} N_{c} F_{1 / 2}(q \phi / k \tilde{T})
\end{aligned}
$$

where $q$ is the electron charge, $\phi$ is the electric potential (related to the conduction band and Fermi level through $-q \phi=E_{C}-E_{F}$ ), and $\rho$ is the free charge distribution, determined by the ionized donor and acceptor concentrations $\left(N_{D}^{+}, N_{A}^{-}\right)$, and electron and hole concentrations $(n, p)$. The free carrier concentrations are then related to the potential through the effective density of states $\left(N_{c}\right)$ and Fermi integral $\left(F_{1 / 2}\right) . k$ is the Boltzmann constant, and here $\tilde{T}$ is temperature.

For a piezoelectric semiconductor, Eqs. 1,2,7-9 are coupled to describe the electro-mechanics of the material. It is important to note that observation of piezoelectric effects in semiconductors relies on the existence of a depletion region, where the semiconductor is not neutral, and therefore is related to junctions and surfaces. Initial observations of coupled behaviour were studied in early and late 1970s reporting photo-mechanical coupling in piezoelectric semiconductors $[7,8]$ and mechanical modulation of metalsemiconductor Schottky contact barrier height [9, 10], later coined as the piezotronic effect $[11,12]$. Lagowski and Gatos described the deformation of CdS and GaAs cantilevers in response to light absorption [7, 8] (Figure 2). They ascribe the observation to generation of surface voltage and converse piezoelectric deformation. This effect was exhibited only when the polar surfaces, [0001] in CdS and [111] in GaAs, were examined (the wide faces of the cantilever). No measurable effect was found for samples with non-polar surfaces, indicating that this effect is related to piezoelectricity. Furthermore, no effect was measured when GaP samples were examined.

Kusaka et al. examined the direct piezoelectric effect, where the Schottky barrier height was measured during bending of metal-semiconductor junctions of CdS and GaP [9, 10]. The Schottky barrier change was monitored through current measurements, and it was found that the polarity of the change corresponded to the crystal face of the Schottky contact, and the material (II-VI and III-V semiconductors' piezoelectric coefficients have opposite signs) - indicating that the effect originated in piezoelectricity. It is interesting to note that $\mathrm{GaP}$ was examined in both experiments, however it 


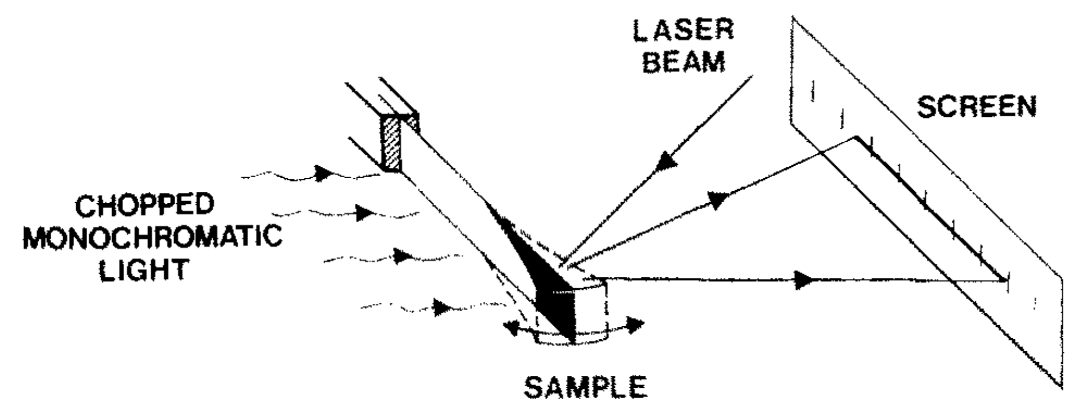

Figure 2: Schematic of the optical setup to measure the photomechanical coupling in piezoelectric semiconducting cantilevers. Reproduced with permission from Elsevier [8].

only exhibited a piezoelectric related effect in the latter - where a device was fabricated. As mentioned by Lagowski and Gatos, this is most likely due to the presence of a surface accumulation layer in free $\mathrm{GaP}$ surfaces, shortening the generated surface photovoltage [8]. This layer is depleted when forming a Schottky contact, allowing for the piezoelectric effect to modify the barrier height.

By solving the piezoelectric equations coupled to Poisson's equation (Eqs. 1,2,79) an expression for the piezotronic effect, the change in barrier height with strain, was presented

$$
\Delta \phi_{B}= \pm \frac{d_{31}}{S_{11}} \cdot \frac{V_{b i}}{q N_{D}} \cdot \frac{1}{R}
$$

where $V_{b i}$ is the built in voltage, and $1 / R$ the curvature of the substrate the piezotronic Schottky diode was mounted on. Figure 3 shows a schematic representation of the piezotronic effect in NWs, as was extensively studied in work from Z.L. Wang's group. Importantly, direct stretching and compressing along the polar axis is more accessible in NWs compared to the bulk.

\section{Why are piezoelectric semiconducting nanowires special?}

This section explores early studies that capture the essence of piezoelectricsemiconductor coupling: interesting phenomena arise in junction regions, where the unique behaviour of semiconductors comes into play. When it comes to semiconductor NWs, their inherent electromechanical characteristics may contribute to an enhanced piezoelectric response, as explained below. 


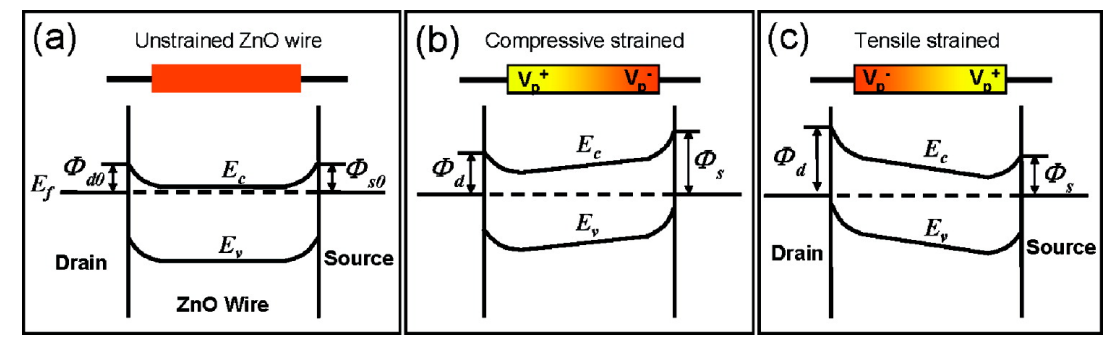

Figure 3: The fields developing within a $\mathrm{ZnO} \mathrm{NW}$ and near the Schottky contacts for a) unstrained; b) compressed; c) stretched NWs. Reproduced with permission from ACS [11].

\subsection{Mechanical properties of piezoelectric semiconducting nanowires}

Nanowires have unique mechanical properties due to their size and slender shape. Nanowires exhibit increased failure stress, compared to known bulk properties. The main reason is the smaller likelihood of structural defects in smaller sizes $[13,14,15]$. For example Wang et al. found that GaAs of $50-150 \mathrm{~nm}$ in diameter could sustain $10-11 \%$ strain before failure, a value significantly larger than measured in bulk material [15]. If so, NWs can withstand higher stresses, which in turn can result in increased piezoelectric response. Furthermore, there are reports of increased stiffness (Young's modulus) in NWs [16]. Revisiting Equation 10 for the piezotronic effect, an increased stiffness (reduced compliance) may result in an increased effect for a given strain.

Due to their aspect ratio, NWs are flexible, unconstrained by their envi-

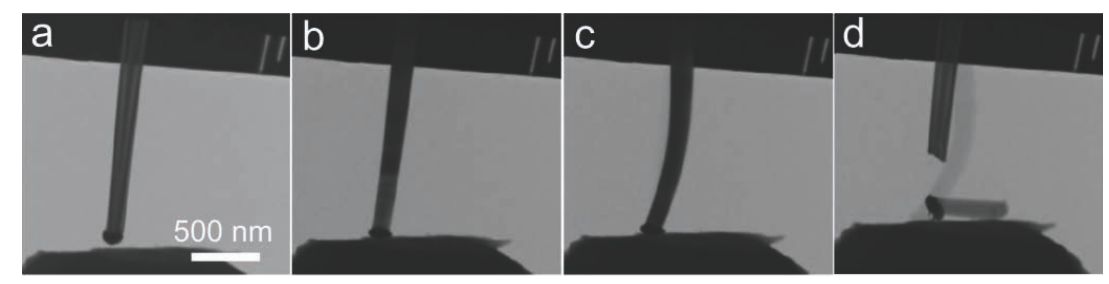

Figure 4: a-d) Different stages of GaAs NW deformation, from contact to failure. Reproduced with permission from Wiley [15].

ronment, and inherently allow larger deformations. Furthermore, they allow the application of tensile and compressive stresses in the same orientation where the electrical properties are examined ( $d_{33}$ type effect) [17]. This is unlike the bulk case, where generally the main deformation axis is orthogonal 
to the examined electrical properties ( $d_{31}$ type effect) - see Figure 5. Generally $d_{33}>d_{31}$, and thus a larger response is to be expected from the former. The increased degree of mechanical freedom comes into play through elimination of substrate boundary condition for the inverse piezoelectric coefficient $[18,19]$

$$
d_{33, b u l k}^{\text {eff }}=d_{33}-\frac{2 S_{13}}{S_{11}+S_{12}} d_{31}
$$

where $d_{33, b u l k}^{e f f}$ is the observed, clamped, coefficient, and $S_{i j}$ are elastic compliance coefficients. For unclamped nanostructures the second term vanishes

$$
d_{33, N W}^{e f f}=d_{33}
$$

Zhao et al. have estimated the (negative) contribution of the substrate to $\mathrm{ZnO}$ piezoelectricity results in about $50 \%$ reduction in the effective piezoelectric coefficient [19].
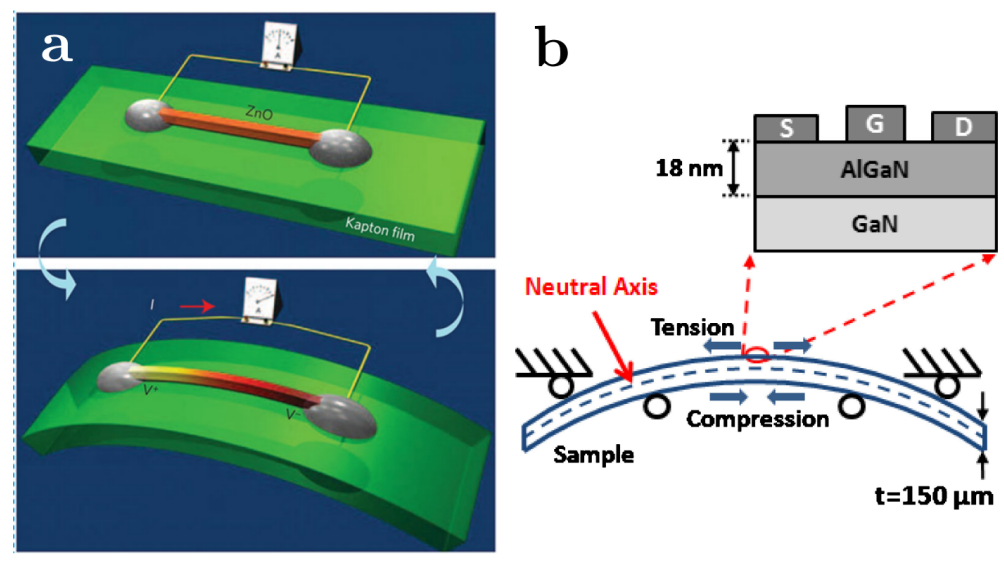

Figure 5: a) Schematic of an axial $\mathrm{ZnO} \mathrm{NW}$ piezoelectric nanogenerator, experiencing axial deformation. Reproduced with permission from Nature Publishing Group [17]; b) Schematic of a GaN/AlGaN HEMT experiencing bending, where the device contacts are transverse to the main strain orientation. Reproduced with permission from Eslsevier [20].

\subsection{Electronic properties of piezoelectric semiconducting nanowires}

Semiconductor NWs have an increased tendency for depletion as their diameter decreases, due to geometry and size related electrostatic effects. Briefly, the cylindrical geometry induces a shallow profile of the potential, 
compared to bulk [21, 22]. When solving Poisson's equation in cylindrical coordinates, considering a depletion region extending from the NW surface towards its center, Eq. 7 takes the form [22]

$$
\frac{d^{2} \phi}{d r^{2}}+\frac{1}{r} \frac{d \phi}{d r}=-\frac{q N_{D}}{\epsilon_{s}} \quad ; \quad R_{d e p}<r<R
$$

where $R$ is the NW radius and $R_{d e p}$ is the onset of the depletion region. Unlike the equation in Cartesian coordinates (used for bulk material), the additional $1 / r$ term (second) requires the first derivative of the potential (i.e., the electric field) to decay slowly in order to avoid singularity - leading to increased depletion.

NW electrostatics also have implications on Schottky barrier height, where non-ideal barrier-lowering effects are weakened in NWs (Figure 6) [23, 24]. A possible implication of increased barrier height and depletion length is reduced screening of the piezoelectric potential formed within NWs in response to strain, leading to a more pronounced piezoelectric response.

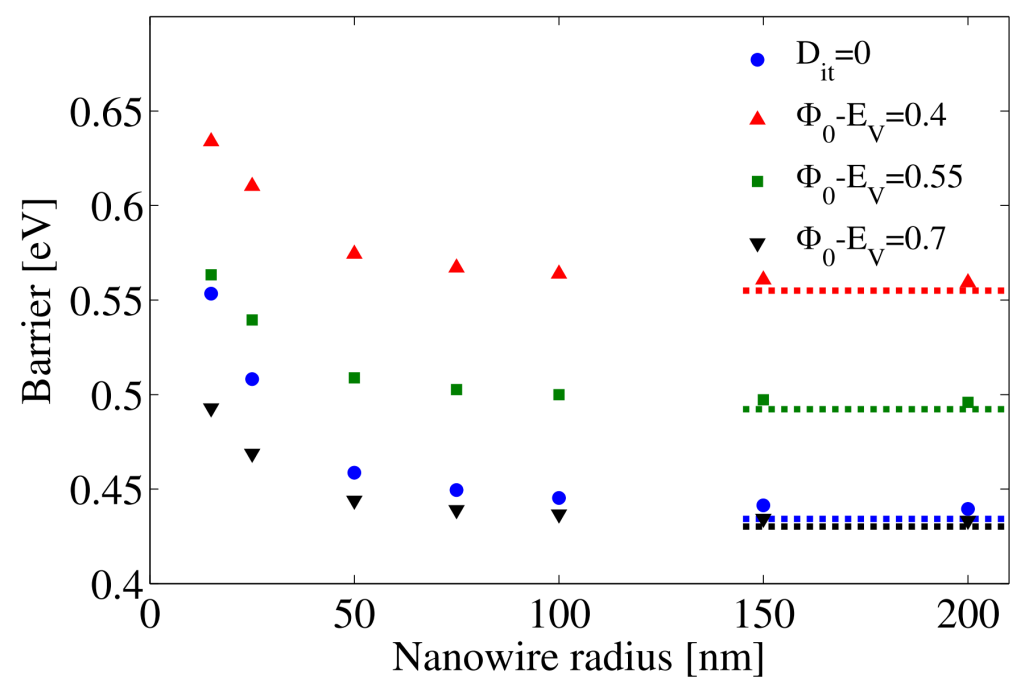

Figure 6: Calculated Schottky barrier height for an N-type semiconducting NW as a function of diameter. Non-ideal effects lowering the barrier become less significant as size is reduced, resulting in a higher barrier for smaller NWs, and increased depletion. The barrier height approaches bulk value (dashed) with size. Reproduced with permission from AIP [24]. 


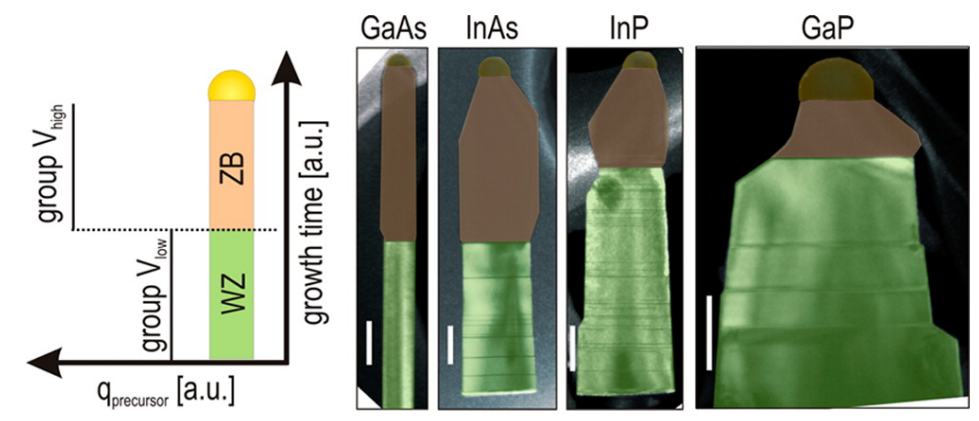

Figure 7: Schematic of the group-V modulation during NW growth (left) resulting in controlled crystal phase switching in several III-V NWs (right). Reproduced with permission from ACS [25].

\subsection{Effect of growth on the piezoelectric properties of semiconducting nanowires}

Semiconductor NWs of the III-V family may crystallize in wurtzite as well as zinc-blende phase, unlike their bulk counterparts which crystallize exclusively as zinc-blende (non-nitride III-Vs)[25]. Figure 7 shows the control achieved in switching growth from zinc-blende to wurtzite structure in InAs, GaAs, InP and GaP NWs. Furthermore, it is possible to control growth orientation to grow NWs where the axial orientation is polar/non-polar. The piezoelectric coefficient matrix (Voigt notation) for wurtzite semiconductors contains three non-degenerate coefficients and is given by

$$
d_{W Z}=\left(\begin{array}{cccccc}
0 & 0 & 0 & 0 & d_{15} & 0 \\
0 & 0 & 0 & d_{15} & 0 & 0 \\
d_{31} & d_{31} & d_{33} & 0 & 0 & 0
\end{array}\right)
$$

This matrix is well known and used, considering the majority of work on piezoelectricity in semiconductors is related to $\mathrm{ZnO}$ and $\mathrm{GaN}$ - both wurtzite materials. It is easily and directly applied to NWs, since the growth orientation of the NW is usually [0001], corresponding to the polar axis (3-axis) of the matrix.

For zinc-blende materials (non-nitride III-Vs) a more complicated picture arises: the piezoelectric matrix is usually presented such that the 3-axis coincides with one of the main cubic axis

$$
d_{Z B,[001]}=\left(\begin{array}{cccccc}
0 & 0 & 0 & d_{14} & 0 & 0 \\
0 & 0 & 0 & 0 & d_{14} & 0 \\
0 & 0 & 0 & 0 & 0 & d_{14}
\end{array}\right)
$$


This matrix has only one non-degenerate coefficient, the shear coefficient $d_{14}$. However, III-V NWs, either crystallizing in wurtzite or zinc-blende, have a tendency to grow in the $[111] /[0001]$ orientation (for zinc-blende/wurtzite correspondingly). Considering the NW symmetry, it makes sense to rotate the piezoelectric matrix in Equation 15 such that the 3-axis is [111]. When done in complete analogy to wurtzite, i.e. the 1-axis corresponding to $[11 \overline{2}] /[10 \overline{1} 0]$ and the 2 -axis corresponds to $[\overline{1} 10] /[\overline{1} 2 \overline{1} 0]$, the matrix takes the following form [26]

$$
d_{Z B,[111]}=\left(\begin{array}{cccccc}
d_{11} & -d_{11} & 0 & 0 & d_{15} & 0 \\
0 & 0 & 0 & d_{15} & 0 & d_{26} \\
d_{31} & d_{31} & d_{33} & 0 & 0 & 0
\end{array}\right)
$$

The distinctions between the piezoelectric matrices of zinc-blende and wurtzite, given by Equations 14 and 16 open up opportunities to engineer the piezoelectric properties of a single NW through formation of crystal-phase heterostructures. For example, there is no radial symmetry in ZB since the response to a field applied along the 1-axis will result in a response different than a field applied along the 2 -axis.

Demonstrating some of the principles mentioned above (Sections 3.1-3.2), even in bulk material, is the work published by Gerngross et al., who measured the piezoelectric response of chemically etched porous InP (zinc blende) [27]. The measured coefficients were found to be up to 30 times those of bulk InP, due to enhanced depletion in the material (increased surface area), and porosity induced softening [28]. On the other extreme, quantum mechanical simulations have predicted two orders of magnitude increase in the piezoelectric coefficients of ultra-thin (1-2 nm) $\mathrm{ZnO}$ and GaN NWs [29].

The piezoelectric properties of $\mathrm{ZnO}$, coupled with the relative ease of fabrication of nanostructures of this material, render it particularly useful and popular for applications in piezoelectric energy harvesting. $\mathrm{ZnO}$ is a direct wide band gap $(\sim 3.3 \mathrm{eV})$ semiconductor with a wurtzite structure, inducing polarity along the c-axis of the structure [30,31,32]. Figure 8a shows the wurtzite lattice structure of $\mathrm{ZnO}$ with $\mathrm{a}=3.25$ and $\mathrm{c}=5.20$, and the origin of the dipole moment that gives rise to piezoelectric in this material [33]. Figure $8 \mathrm{~b}$ illustrates a typical growth morphology of $\mathrm{ZnO}$ nanowire or nanorod with a hexagonal crystal structure and the corresponding facets, where (001) 
is the polar surface and relatively more reactive while the side facets are non-polar and more stable [30, 31, 32]. Therefore, the $\mathrm{ZnO}$ crystal tends to grow faster along c-axis, which is favourable particularly during nanowire growth as it results in a net dipole moment along the axis of the nanowire. $\mathrm{ZnO}$ has been shown to be easily grown into a variety of nanostructures with different morphologies and shapes, such as nanorods [31, 34, 35], nanotubes $[36,37]$, nanowires [38, 31, 39, 40], nanobelts [41] and nanorings, onto a variety of different substrates at relatively low temperatures $\left(<100^{\circ} \mathrm{C}\right)$ compared to high-temperature-sintered piezoceramics.

Commonly used synthesis techniques include chemical vapour deposition [42], physical vapour deposition [30, 43], sol-gel synthesis [44, 45] electrodeposition $[45,46,47,48]$ and hydrothermal synthesis $[31,32,49,50,51,52,53]$. $\mathrm{ZnO}$ bulk and thin films are likely to suffer from poor piezoelectric performance due to defects both in the bulk and on the surface that may arise due to environmental degradation $[38,54,55]$, while the relatively high stiffness constant of $\mathrm{ZnO}$ renders it prone to mechanical failure [56]. However, these limitations of $\mathrm{ZnO}$ could be overcome by incorporating nanostructures into nanocomposite systems, as nanostructured $\mathrm{ZnO}$ with higher aspect ratio and surface-to-volume ratio, possesses enhanced sensitivity to low-amplitude ambient vibrations as well as reduced fragility compared with their bulk or thin film counterparts $[57,58]$, making them particularly attractive for energy harvesting applications. In general, single crystal NWs are preferred over polycrystalline ones because there is a single domain of electric dipoles with one polarization direction, but even polycrystalline $\mathrm{ZnO}$ nanowires with preferred orientation along the nanowire axis have been shown to exhibit robust piezoelectric energy harvesting performance [57, 59].

\section{Characterization of Piezoelectricity in Semiconducting Nanowires}

Traditional macro-scale characterization of piezoelectricity is usually done by optical methods (see Figures 9a and 2) for displacement measurement. However these methods are hardly applicable when considering nanostructures such as NWs, which are smaller than the characteristic wavelength. The prevalent tool for nanoscale piezoelectric characterization is piezoresponse force microscopy (PFM), which has been widely used for characterization of ferroelectric and piezoelectric materials [60]. However, when it comes to NWs, either vertical or horizontal, the application of PFM is challenging, due 

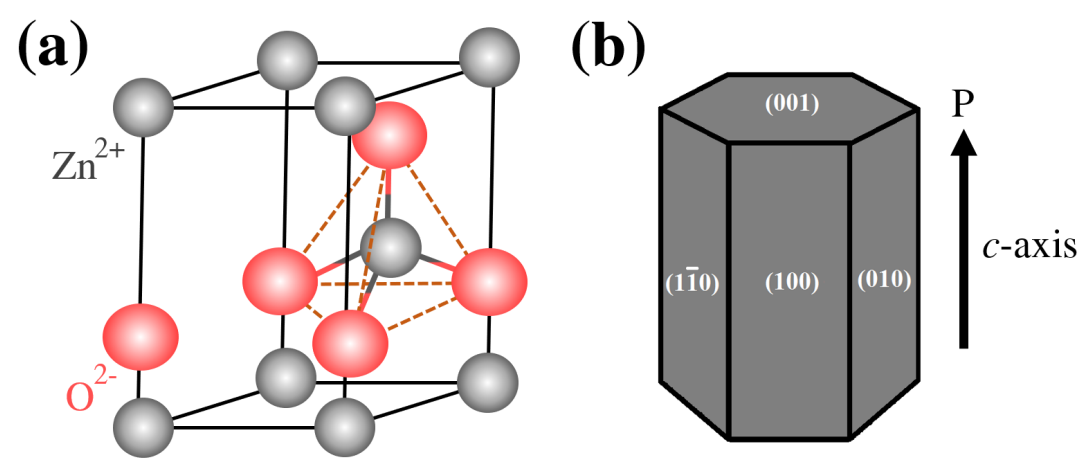

Figure 8: a) Wurtzite structure model of $\mathrm{ZnO}$; b) Typical growth morphology of $\mathrm{ZnO}$ with hexagonal crystal structure. The arrow indicates the polarization direction and the c-axis direction.

to possible mechanical damage and/or dislodgment during contact-mode operation, and reports of PFM-based NW characterization are not common. Other methods such as atomic force microscopy (AFM) based current generation or characterization of the direct piezoelectric effect, are usually applied to NWs. These are discussed in the following sections:

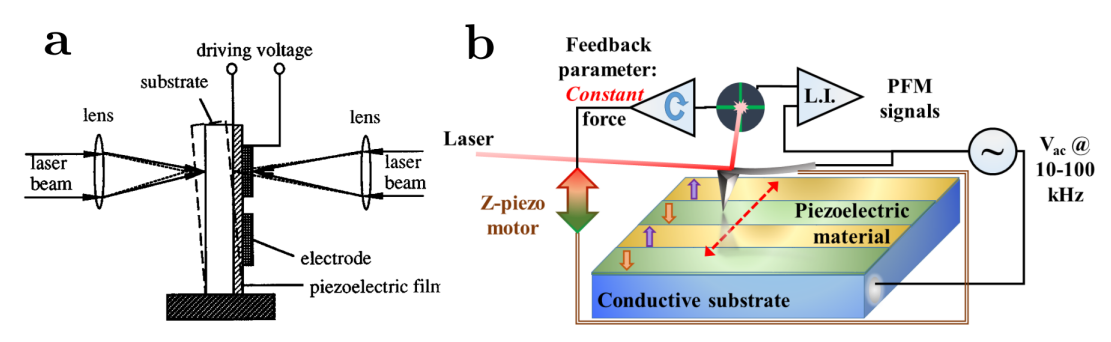

Figure 9: a) Schematic of a dual beam interferometer used to measure bulk material piezoelectric effects. Reproduced with permission from AIP [61]; b) Schematic of a PFM setup: a conductive tip applies AC voltage to a piezoelectric sample, and simultaneously follows the resulting deformation. Reproduced with permission from The Royal Society of Chemistry [62].

\subsection{Piezoresponse Force Microscopy}

PFM works on the basis of the converse piezoelectric effect: a conductive AFM tip is scanned across a piezoelectric sample in contact mode, while an 
AC voltage is applied between the tip and the bottom electrode, through the sample. In response to the bias the sample deforms, and the deformation is monitored through a laser spot reflection from the tip, maintaining a constant contact with the sample through the feedback (Figure 9b). Due to contact mode operation, application of PFM to nanostructures is challenging, and advanced PFM methods are be required for characterization[26], as discussed below in detail.

The first report of PFM application to semiconductor nanostructures was published by Zhao et al., describing the measurement of piezoelectricity in $\mathrm{ZnO}$ nanobelts [19]. It was found that the piezoresponse of the nanobelt is 2-3 times stronger than that of bulk material. This finding was explained by the unconstrained boundaries of the nanobelt (Eqs. 11-12).

Most PFM work revolved around characterization of relatively thick, vertical, NWs - predominantly $\mathrm{ZnO}$ and GaN [63, 35, 64, 65]. Scrymgeour and Hsu have correlated PFM and conductive AFM (c-AFM) measurements of $\mathrm{ZnO}$ nanorods (Figure 10). Interestingly, they have found that thinner nanorods $(\sim 150 \mathrm{~nm})$ yield higher piezoelectric responses, and these are coupled to lower conductivity, while the opposite is true for thicker nanorods $(\sim 500 \mathrm{~nm})$. The size dependence could be explained by the increased depletion tendency of thin NWs, as discussed in section 3.2. In is noteworthy that the NWs in this study underwent an annealing treatment to form ohmic contacts, and therefore the more common piezotronic effect is not observed in this case. Furthermore, the NWs were mechanically protected by embedment in a polymer layer, probably to overcome contact-PFM induced mechanical damage for non embedded NWs.

Reports of PFM measurements on horizontal NWs are scarce, mainly due to the challenge of using contact mode PFM on unconstrained objects. In a study by Minary-Jolandan et al. horizontal GaN NWs were studied by PFM. To avoid mechanical damage, the NWs were clamped to the surface at the edges [66]. In this work two configurations were used to realize characterization of the three non-degenerate piezoelectric coefficients of the wurtzite structure - $d_{33}, d_{31}$ and $d_{15}$. For a horizontal NW in a standard PFM configuration, where the voltage is applied across the NW axis, the only measurable deformation is related to $d_{15}$, through a lateral signal (Figure 11b). Characterizing $d_{33}$ and $d_{31}$ requires applying voltage along the NW axis (assuming it is in [0001] orientation), and therefore should be done by applying the signal through lateral electrodes to the NW (Figure 11a). The results ob- 

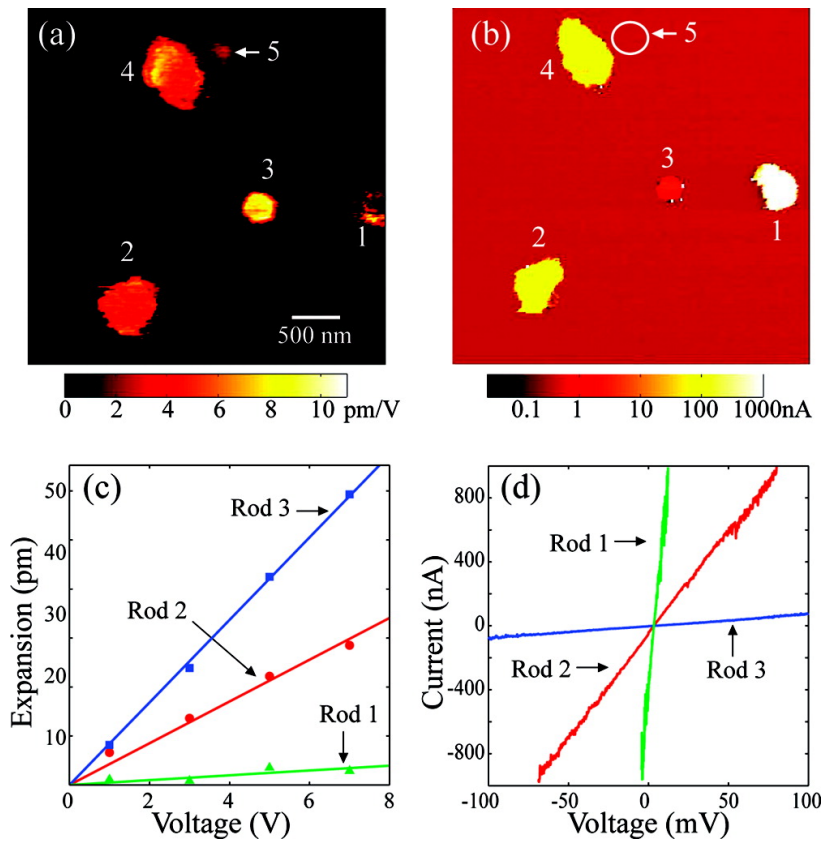

Figure 10: a) PFM and b) c-AFM signals from the same area; c) PFM expansion and d) measured current from the NW shown in $(a, b)$. Reproduced with permission from ACS $[35]$.

tained by Minary-Jolandan et al. for the piezoelectric coefficients, $d_{33}=12.8$, $d_{31}=-8.2$ and $d_{15}=10.2 \mathrm{pm} / \mathrm{V}$, are $3-4$ times higher than accepted bulk values [66].

Recently, the problems related to contact mode PFM characterization of nanomaterials have been recognized, and non-destructive PFM methods combining mechanical indentation modes with piezoresponse analysis have been developed (Figure 12) [62, 67]. At the heart of these methods lie two principles: i) minimizing the contact-mode mechanical damage to the sample using an intermittent contact mode; ii) extraction of the PFM data from the period of time were significant tip/sample contact is achieved. using ND-PFM the piezoelectric properties of non-clamped horizontal GaAs NWs were examined, as well as vertical InP NWs. This was the first direct PFM measurement of the $d_{33}$ value of wurtzite InP. The value obtained, $\sim 1 \mathrm{pm} / \mathrm{V}$, was in the center of the theoretical predicted range of values [26].

ND-PFM was further employed to characterize VLS grown horizontal 


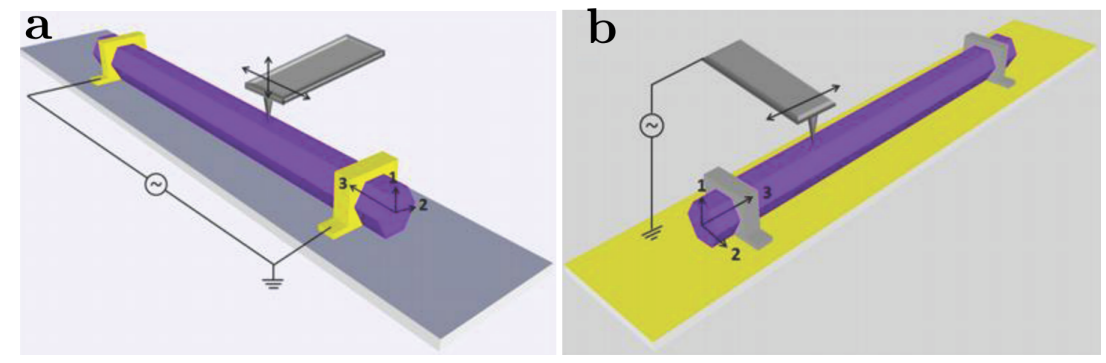

Figure 11: Two configurations for complete characterization of piezoelectricity in horizontal GaN NWs: a) voltage is applied along the NW [0001] axis for $d_{33}$ and $d_{31}$ measurement; b) voltage is applied across the NW axis to measure $d_{15}$. Reproduced with permission from ACS [66].

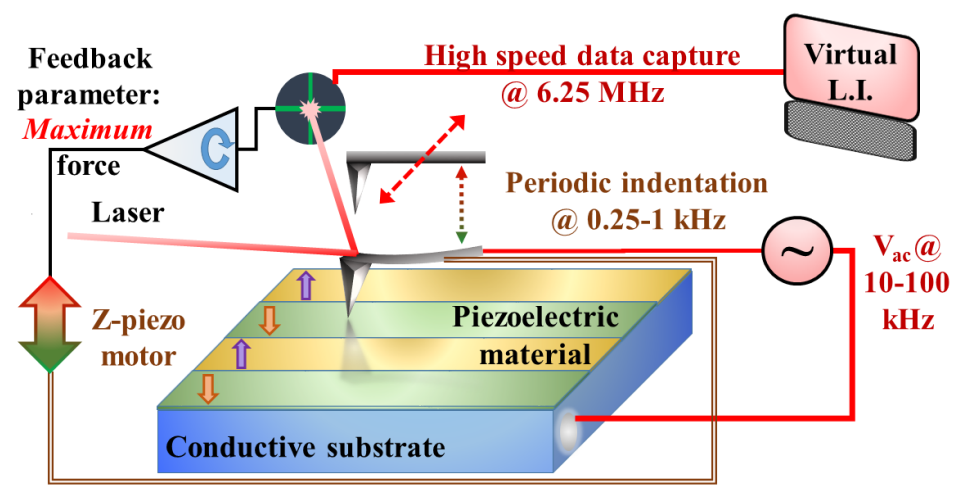

Figure 12: Schematic of the ND-PFM setup: the tip periodically indents the sample, minimizing shear stress damage. An AC voltage is applied to induce the converse piezoelectric effect, and the piezoresponse is then extracted from the time periods the tip is in contact with the sample. Reproduced with permission from The Royal Society of Chemistry [62].

GaAs NWs with a predominant zinc-blende phase [26], and electrodeposited $\mathrm{ZnO}$ NWs [57]. Both materials allowed examination of the material in uncommon configurations: for GaAs - due to the horizontal configuration, and for $\mathrm{ZnO}$ - due to predominant polycrystalline nature, and a non-polar oriented NW axis. ND-PFM provided insight into intricacies of nanoscale piezoresponse, unavailable by contact PFM, which caused sample damage. In the case of GaAs NWs, the results were found to coincide with finite element simulations for the vertical and lateral deflections of the AFM due to the piezoelectric response of the NW, demonstrating the distinct contributions of different piezoelectric coefficients and side facets (Figure 13c,d). For ZnO, a piezoresponse matching the polycrystalline nature of the sample was found 
(Figure 13f).

\subsection{Conductive AFM based measurements}

A significant portion of piezoelectric NW characterization was performed using conductive-AFM techniques [35, 68, 69, 70, 71, 72, 73]. Generally, the tip is scanned or indented over vertically aligned NWs, grown on a conductive substrate (Figure 14). Since current conduction takes place in this setup, electrical contacts to the NWs play a significant role in understanding the measurement results, and often there is a Schottky contact between the NW and the conductive AFM tip. As shown in Figure 14 as the tip is scanned across the tip of the vertical NW, one side of the NW is strained while the other is compressed. The direction of the generated piezoelectric voltage is opposite in the two cases. Due to the piezotronic effect, in one instance the Schottky barrier between the NW and tip is lowered, while in the other it is increased; correspondingly, in the first case current flow (piezo-generated) is more efficient, 

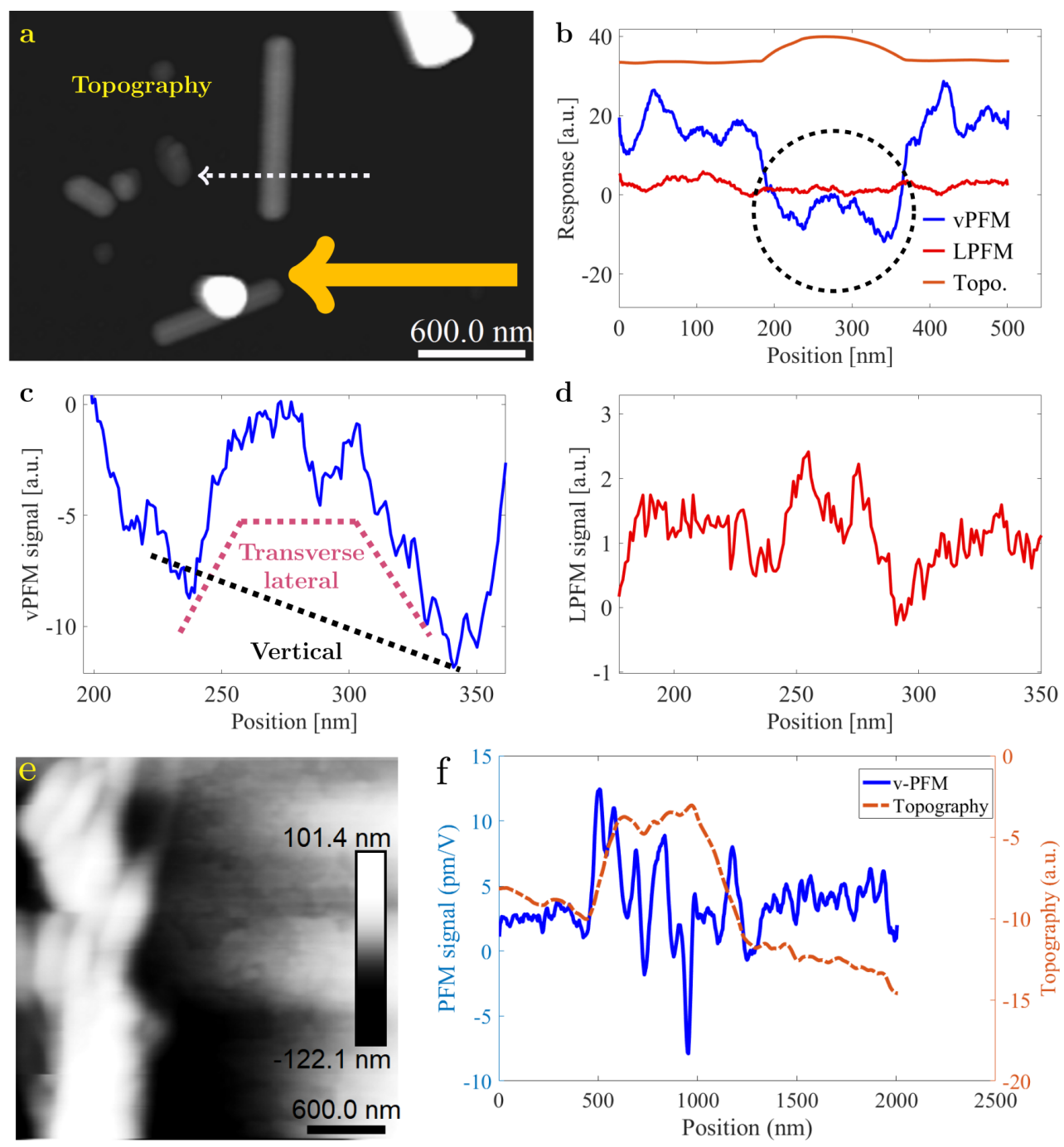

Figure 13: ND-PFM measurements of a GaAs (a-d) and ZnO (e-f) NWs, taken across the NWs: a) GaAs NW topography, with scan direction and cantilever orientation (dashed and solid arrows correspondingly); b) PFM results, showing the vertical and lateral signals, alongside the scan topography. The dashed circle indicates the area of interest; c) close-up to the vertical PFM signal, the dashed lines indicate the simulated signals which may explain the experimental results; d) close-up to the lateral PFM signal, showing the signature of the side facets. Reproduced with permission from IOP [26]; e) ZnO polycrystalline NW topography; f) calibrated vertical PFM response from the NW, showing regions of \pm $10 \mathrm{pm} / \mathrm{V}$, in agreement with the poly-crystallinity and non-polar growth orientation. 
while in the latter, significantly less current is measured. Lu et al. have measured current generation from both n- and p-type ZnO NWs. It was found that the measured voltage in the two cases is of opposite polarity, and that it originates from different sides of the NW (strained/compressed) - in accordance with the explanation that the piezotronic effect dominates the measurement.
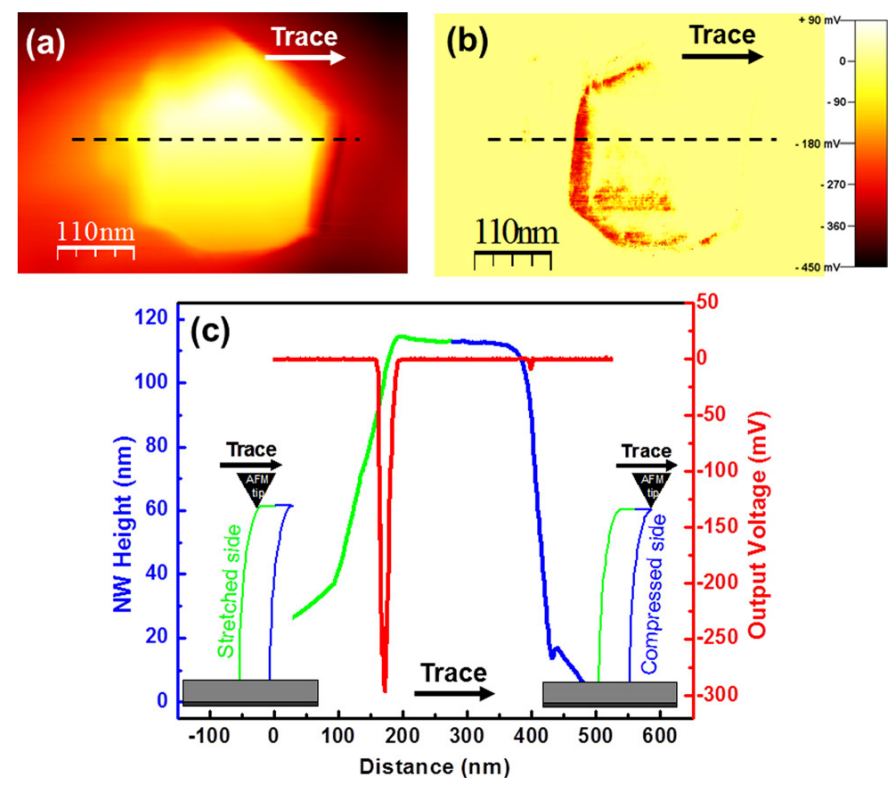

Figure 14: Current asymmetry in the cAFM measurements of a GaN NW a) NW topography; b) current map, showing the current arising in one side; c) line scan of the current map and topography showing the current arising on the stretched side of the NW, and subsiding on the compressed side. Reproduced with permission from AIP [72].

Wang et al. have examined the influence of doping concentration in the range of $10^{17}-10^{19} \mathrm{~cm}^{-3}$ on the performance of NW based nanogenerators and piezotronic junctions [74]. They have found that for nanogenerator operation, where there is a need to generate voltage and induce current flow, output current voltage was not a monotonic function of the doping, but that there is an optimal value of doping mitigating the decrease in resistivity on the one hand side, and screening of the piezoelectric potential on the other. For piezotronic applications, where the main effect is changing the Schottky barrier height, they have found that the lowest concentration yields the highest electromechanical gauge factor. This result stems from the increased 
depletion region associated with lower doping levels. A notable distinction between this method and PFM is the need to generate significant stresses (by bending), while due to the AFM deflection sensitivity (pm range) horizontal NWs can be examined as well as vertical.

\subsection{Directly measured piezoelectric properties of semiconducting nanowires}

Both AFM based methods open up an avenue for basic studies of NW piezoelectricity, however, the motivation for piezoelectric work on semiconductor NWs is mechanical energy harvesting and piezo-photo-tronic applications, and direct piezoelectric performance was frequently examined. Two configurations are usually considered: vertical (Figure 15a), where the NWs are compressed and released to excite the piezoelectricity[73, 75, 76, 77], and horizontal (Figure 15b), where the NWs are lying on a flexible substrate and are compressed/strained in accordance with the substrate [17, 76, 78]. Notably, in most of these cases, the NW experiences uniform strain/compression, unlike the conductive AFM case, where the NW experiences nearly pure bending. Furthermore, through rational design of growth and contacting, enhancement of the output voltage from $\mathrm{mV}$ to $\mathrm{V}$ range (serial connection of lateral devices), and current from $\mathrm{nA}$ to $\mu \mathrm{A}$ range (vertical array devices) was achieved.
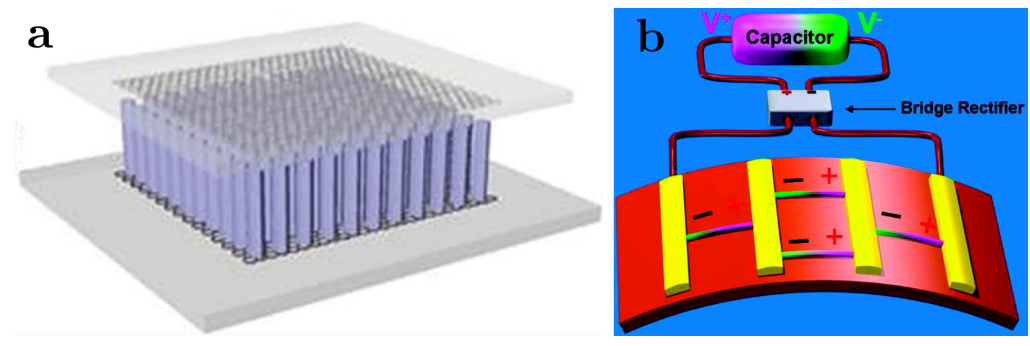

Figure 15: Connecting NWs for energy applications. a) parallel connections of a NW array, maximizing generated current. Adapted with permission from Wiley [77]; b) serial connection of NWs, maximizing generated voltage. Adapted with permission from ACS [78]

The potential of piezoelectric semiconductor NWs for realizing further advanced applications such as piezoelectric modulated field effect transistors (FETs) [79, 80], or piezotronic logic [81], have also been demonstrated. 
Kwon et al. have shown that through piezotronic modulation of the gateoxide-semiconductor junction, the effective carrier mobility increases 4 times for $1.5 \%$ strain of the NW FET. They have ruled out piezoresistive effects by demonstrating an opposite effect on top- and bottom-gated devices, indicating that the crystal orientations determines the performance, and not the strain in-itself. Wu et al. used horizontal $\mathrm{ZnO}$ NWs located on both sides of a flexible substrate to realize a logic inverter [81]. Bending the substrate results in compression of NWs on one side, and strain of the NW on the other. Due to the piezotronic effect, one Schottky barrier is reduce while the other increased leading to inverter operation (Figure 16).

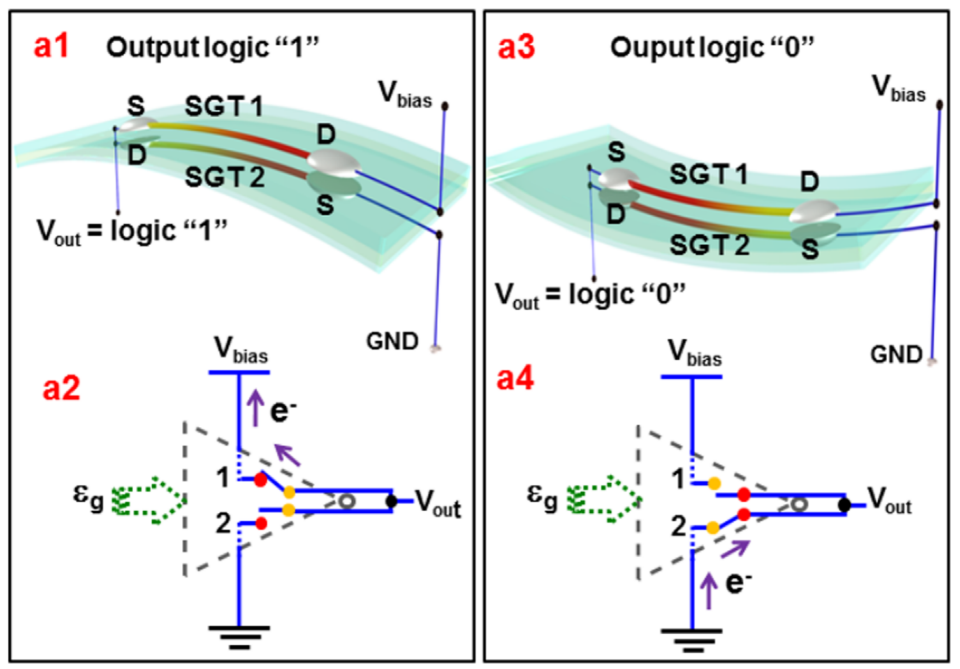

Figure 16: Schematic of the two sided $\mathrm{ZnO}$ NW based logic. The complementary response of the NWs on the opposite sides of the substrate, allows realizing an intverter operation. Reproduced with permission from Wiley [81].

\section{Piezoelectricity in Semiconductor Nanowires for Energy Appli- cations}

\subsection{II-VI Nanowires}

Owing to a variety in synthesis methods [82, 83] and outputs [82, 84] (NWs, nanoribbons) $\mathrm{ZnO}$ was the first semiconductor nanostructure to be vastly studied for nanoscale piezoelectric applications [85, 86]. Other members of the II-VI family such as CdSe, CdS, and ZnS were examined as 
well [87, 88, 89]. These materials generally have large, direct, band-gaps, and higher piezoelectric coefficients compared to III-Vs (also of opposite sign) [90, 91], making them attractive candidates for piezotronic applications.

\subsection{1. $\mathrm{ZnO} \mathrm{NWs}$}

$\mathrm{ZnO} \mathrm{NWs}$ have been widely reported for their use in various energy applications, including as piezoelectric nanogenerators for mechanical energy harvesting. ZnO possesses good piezoelectric properties at nanoscale [92, 93], which in some cases can be comparable to lead-free piezoceramic oxides. Other attractive properties include ease of nano-synthesis [57], being environment friendly [94] biocompatible [95], lightweight [96], scalable and low-cost [97] with good mechanical [98] and thermal stability [44]. In most cases, ZnO nanowires are grown on a conducting substrate which serves as the bottom electrode, and a top electrode is separately applied post-growth. The device is then subjected to mechanical excitation and the resulting piezo-generated charge is collected to drive an external circuit. The presence of a substrate may introduce issues related to flexibility of the device and/or delamination of NWs during operation. To overcome this issue, $\mathrm{ZnO}$ nanowire-based nanogenerators have been developed where a polymer is introduced into the device design to improve mechanical stability as well as flexibility. Such device designs include dispersions of $\mathrm{ZnO}$ NWs within a soft polymeric matrix, however, this has the disadvantage of randomised polarization directions of the individual $\mathrm{ZnO} \mathrm{NWs}$. As a matter of fact, fatigue performance of $\mathrm{ZnO}$ based nanogenerators is often not reported in the literature, as the material is additionally prone to environmental and mechanical degradation over time. However, studies where $\mathrm{ZnO}$ nanowires have been grown within nanoporous polycarbonate templates, and then incorporated into nanogenerators while still embedded and aligned, have resulted in long and reliable energy harvesting device lifetimes [57, 59]. Table 1 summarizes key ZnO nanowire-based nanogenerators that have been reported over the years, highlighting inherent advantages and disadvantages of each.

\subsubsection{Other II-VIs}

An early demonstration of piezoelectric current generation from a 150-100 nm CdS NWs was published by Lin et al.in 2008[87]. Using the conductive AFM method, different responses were achieved on the stretched and compressed sides of the NW, confirming piezoelectric dominated behaviour. The output current was found to increase when pure WZ NWs were examined, 


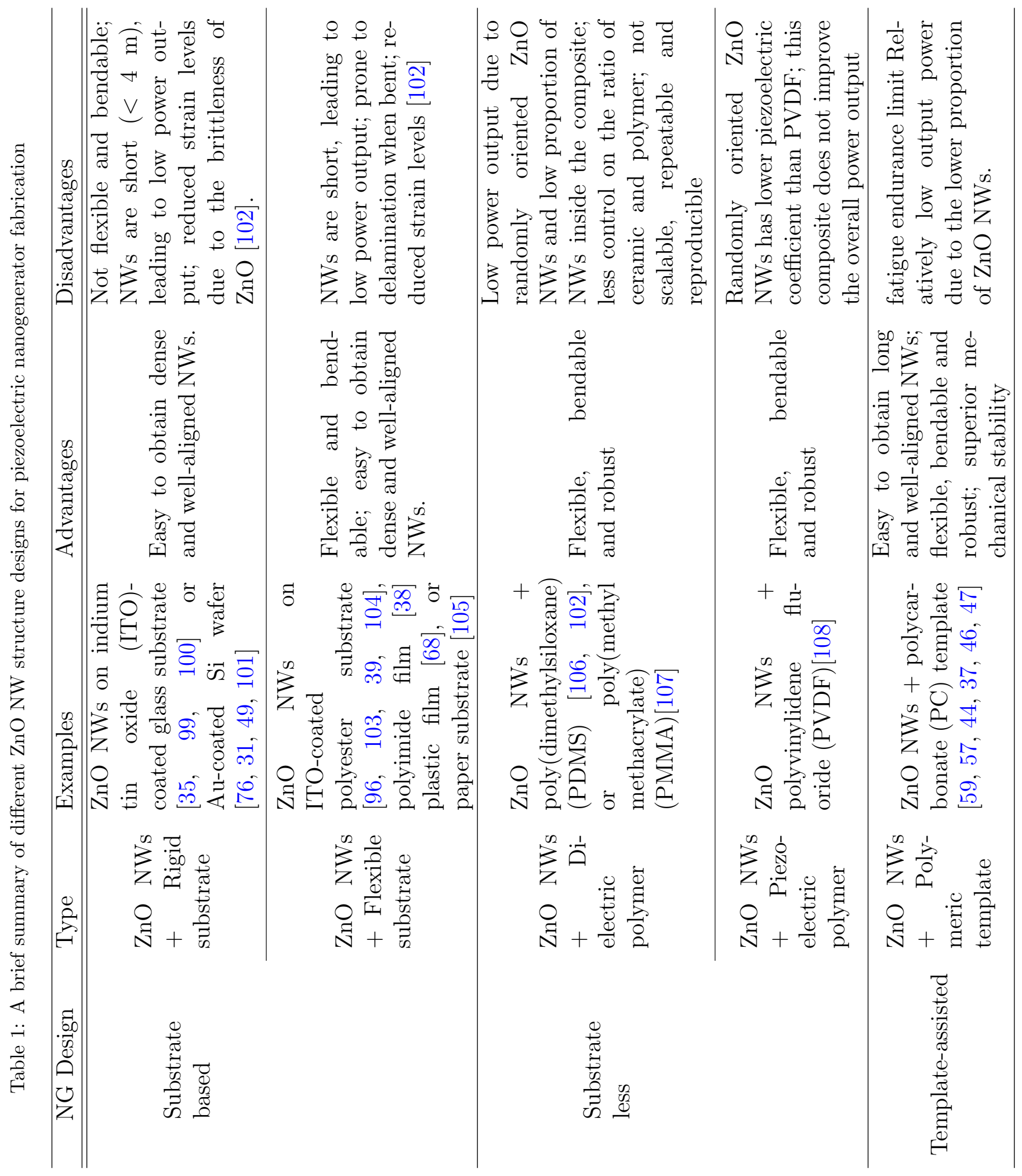


compared to $\mathrm{WZ} / \mathrm{ZB}$ mixed phase NWs. In a later work, the piezotronic effect was also demonstrated on CdS and CdSe NWs [109, 110], and energy harvesting with CdTe NWs [111].

\subsection{III-V Nanowires}

\subsubsection{III-Nitride Nanowires}

Alongside $\mathrm{ZnO}$, III-Ns, and GaN in particular is the most widely studied piezoelectric NW material. The piezoelectric coefficients of GaN and AlN (about 3 and $5 \mathrm{pm} / \mathrm{V}$ [112]) are smaller than those of $\mathrm{ZnO}(10-12 \mathrm{pm} / \mathrm{V})$, thus making them less attractive for energy harvesting applications. However the large range of visible range band-gap tunability provided by III-N alloying, suggests that combining piezotronic and photonic applications - coined as piezo-phototronics is worthwhile. The effect of strain on the performance of III-N based optical devices has been examined [113, 114, 115, 116]. As mentioned above (Section 2.2), polarization induced internal fields result in reduced emission efficiency. Peng et al. have demonstrated the control of InGaN/GaN multi-quantum well NW LED by application of stress [113]. Subsequently, strain has been shown to improve the emission of bulk InGaN/GaN based LEDs [117].

Mechanical energy harvesting has also been successfully demonstrated using various types of III-N NWs, exploring issues such as doping and geometrical design and their influence on energy harvesting efficiency.[118, 119, $71,73,74,120]$.

\subsubsection{Non-nitride III-V Nanowires}

The piezoelectric properties of III-As and III-P have not received significant attention, despite being known and utilized for electro-mechanical applications [121, 122]. The main reasons for that are the lower piezoelectric coefficients, and non-polar technology ([001] and [110] cubic orientations in zinc-blende), prevailing non-nitride III-V bulk electronic and optoelectronic technology [123, 5]. Nonetheless, the various nanostructures Vs. bulk distinctions described in Section 3, apply to non-nitride III-Vs as well. Indeed high piezoelectric coefficients have been predicted for III-Vs in the wurtzite phase [124], which is relevant for III-V NWs. Except from PFM work mentioned above[26], piezoelectric generation from III-V were examined [125, 126], pointing out the role of stress distribution from an upper contact to a single NW piezo-element, in increasing the piezoelectric efficiency. Recently, the piezoelectric properties of wurtzite GaAs were examined using 


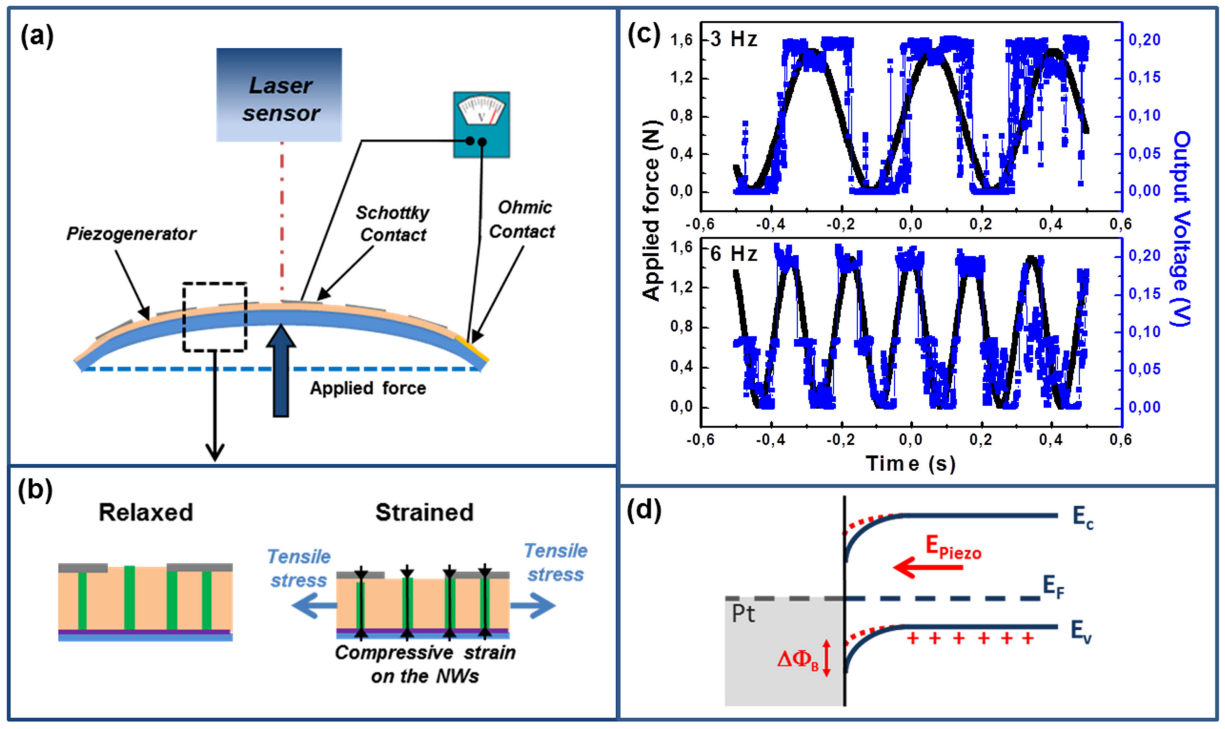

Figure 17: Schematic and operation of a GaN array based nanogenerator. a) schematic of the device and experimental setup; b) schematic of the mode of operation, note that the NWs are compressed through transverse stretching; c) nanogenerator output voltage; d) schematic band diagram for the Schottky contact, which is responsible for the effect through modulation of the charges in the depletion region. Reproduced with permission from IOP [73].

the c-AFM apparatus [127], demonstrating the piezo-phototronic effect in increasing generated current under illumination.

\section{Summary}

The piezoelectric properties of semiconducting nanowires have many applications in energy harvesting and sensing devices based on the inter-dependence of the mechanical, electrical and optical properties of these materials. In most cases, nanowires have been found to exhibit superior electromechanical properties when compared to the bulk of the material, and these can be further tuned by controlling the growth method. The crystalline phases present in these semiconducting nanowires play a strong role in determining the nature of piezoelectricity in these systems, and hence advances in growth and characterization of these nanowires have paved the way for the development of novel devices based on this effect. Importantly, a greater level of understanding and control of piezoelectric properties of semiconducting nanowires 
will serve to underpin advances in the emerging fields of piezotronics and photo-piezotronics, as well as in the development of energy harvesters for small-power applications. Materials and device engineering at the nanoscale therefore offer new routes towards exploiting the piezoelectric properties of semiconducting nanowires for a wide range of energy applications, and there remains plenty of scope for innovation in this area. 


\section{Bibliography}

[1] Jacques Curie and Pierre Curie. Phénomènes électriques des cristaux hémièdres à faces inclinées. Journal de Physique theorique et appliquee, 1(1):245-251, 1882.

[2] Junmin Hu, RG Beck, Tao Deng, RM Westervelt, KD Maranowski, AC Gossard, and George M Whitesides. Using soft lithography to fabricate GaAs/AlGaAs heterostructure field effect transistors. Applied physics letters, 71(14):2020-2022, 1997.

[3] O Ambacher, J Smart, JR Shealy, NG Weimann, K Chu, M Murphy, WJ Schaff, LF Eastman, R Dimitrov, L Wittmer, et al. Twodimensional electron gases induced by spontaneous and piezoelectric polarization charges in N-and Ga-face AlGaN/GaN heterostructures. Journal of applied physics, 85(6):3222-3233, 1999.

[4] Min-Ho Kim, Martin F Schubert, Qi Dai, Jong Kyu Kim, E Fred Schubert, Joachim Piprek, and Yongjo Park. Origin of efficiency droop in GaN-based light-emitting diodes. Applied Physics Letters, 91(18):183507, 2007.

[5] Colin Wood and Debdeep Jena. Polarization effects in semiconductors: from ab initio theory to device applications. Springer Science \& Business Media, 2007.

[6] Simon M Sze and Kwok K Ng. Physics of semiconductor devices. John wiley \& sons, 2006.

[7] J Łagowski and HC Gatos. Photomechanical Effect in Noncentrosymmetric Semiconductors-CdS. Applied Physics Letters, 20(1):14-16, 1972 .

[8] Jacek Eagowski and Harry C Gatos. Photomechanical vibration of thin crystals of polar semiconductors. Surface Science, 45(2):353-370, 1974.

[9] Masahiko Kusaka, Masaaki Kanakura, and Susumu Okazaki. Interface Properties and Schottky Barrier on the Polar Surfaces of CdS and GaP Crystals. Japanese Journal of Applied Physics, 13(S2):437, 1974.

[10] M Kusaka. Electrical properties of metal-piezoelectric semiconductor interface under stress. Surface Science, 78(1):209-219, 1978. 
[11] Jun Zhou, Peng Fei, Yudong Gu, Wenjie Mai, Yifan Gao, Rusen Yang, Gang Bao, and Zhong Lin Wang. Piezoelectric-potential-controlled polarity-reversible Schottky diodes and switches of $\mathrm{ZnO}$ wires. Nano letters, 8(11):3973-3977, 2008.

[12] Zhong Lin Wang. Nanopiezotronics. Advanced Materials, 19(6):889892, 2007.

[13] Baomei Wen, John E Sader, and John J Boland. Mechanical properties of $\mathrm{ZnO}$ nanowires. Physical Review Letters, 101(17):175502, 2008.

[14] Michael J Gordon, Thierry Baron, Florian Dhalluin, Pascal Gentile, and Pierre Ferret. Size effects in mechanical deformation and fracture of cantilevered silicon nanowires. Nano letters, 9(2):525-529, 2009.

[15] Yan-Bo Wang, Li-Feng Wang, Hannah J Joyce, Qiang Gao, Xiao-Zhou Liao, Yiu-Wing Mai, Hoe H Tan, Jin Zou, Simon P Ringer, HuaJian Gao, et al. Super deformability and Youngs modulus of GaAs nanowires. Advanced Materials, 23(11):1356-1360, 2011.

[16] Ravi Agrawal, Bei Peng, Eleftherios E Gdoutos, and Horacio D Espinosa. Elasticity size effects in $\mathrm{ZnO}$ nanowires- a combined experimental-computational approach. Nano letters, 8(11):3668-3674, 2008.

[17] Rusen Yang, Yong Qin, Liming Dai, and Zhong Lin Wang. Power generation with laterally packaged piezoelectric fine wires. Nature nanotechnology, 4(1):34-39, 2009.

[18] K Lefki and GJM Dormans. Measurement of piezoelectric coefficients of ferroelectric thin films. Journal of applied physics, 76(3):1764-1767, 1994.

[19] Min-Hua Zhao, Zhong-Lin Wang, and Scott X Mao. Piezoelectric characterization of individual zinc oxide nanobelt probed by piezoresponse force microscope. Nano Letters, 4(4):587-590, 2004.

[20] M Stutzmann, G Steinhoff, M Eickhoff, O Ambacher, CE Nebel, J Schalwig, R Neuberger, and G Müller. GaN-based heterostructures for sensor applications. Diamond and related materials, 11(3):886-891, 2002. 
[21] BS Simpkins, MA Mastro, CR Eddy Jr, and PE Pehrsson. Surface depletion effects in semiconducting nanowires. Journal of Applied Physics, 103(10):104313, 2008.

[22] Yonatan Calahorra and Dan Ritter. Surface depletion effects in semiconducting nanowires having a non-uniform radial doping profile. Journal of Applied Physics, 114(12):124310, 2013.

[23] Yonatan Calahorra, Dan Mendels, and Ariel Epstein. Rigorous analysis of image force barrier lowering in bounded geometries: application to semiconducting nanowires. Nanotechnology, 25(14):145203, 2014.

[24] Yonatan Calahorra, Eilam Yalon, and Dan Ritter. On the diameter dependence of metal-nanowire schottky barrier height. Journal of Applied Physics, 117(3):034308, 2015.

[25] Sebastian Lehmann, Jesper Wallentin, Daniel Jacobsson, Knut Deppert, and Kimberly A Dick. A general approach for sharp crystal phase switching in InAs, GaAs, InP, and GaP nanowires using only group V flow. Nano letters, 13(9):4099-4105, 2013.

[26] Yonatan Calahorra, Xin Guan, Nripendra N Halder, Michael Smith, Shimon Cohen, Dan Ritter, Jose Penuelas, and Sohini Kar-Narayan. Exploring piezoelectric properties of III-V nanowires using piezoresponse force microscopy. Semiconductor Science and Technology, 32(7):074006, 2017.

[27] Mark-Daniel Gerngross, Veaceslav Sprincean, Malte Leisner, Jürgen Carstensen, Helmut Föll, and Ion Tiginyanu. Porous InP as Piezoelectric Component in Magneto-Electric Composite Sensors. ECS Transactions, 35(8):67-72, 2011.

[28] SeungNam Cha, Seong Min Kim, HyunJin Kim, JiYeon Ku, Jung Inn Sohn, Young Jun Park, Byong Gwon Song, Myoung Hoon Jung, Eun Kyung Lee, Byoung Lyong Choi, et al. Porous PVDF as effective sonic wave driven nanogenerators. Nano letters, 11(12):5142-5147, 2011.

[29] Ravi Agrawal and Horacio D Espinosa. Giant piezoelectric size effects in zinc oxide and gallium nitride nanowires. A first principles investigation. Nano letters, 11(2):786-790, 2011. 
[30] Zhong Lin Wang. Nanogenerators for self-powered devices and systems. Georgia Institute of Technology, SMARTech digital repository (http://hdl.handle.net/1853/39262), 2011.

[31] Lionel Vayssieres. Growth of arrayed nanorods and nanowires of $\mathrm{ZnO}$ from aqueous solutions. Advanced Materials, 15(5):464-466, 2003.

[32] Sheng Xu and Zhong Lin Wang. One-dimensional ZnO nanostructures: Solution growth and functional properties. Nano Research, 4(11):10131098, 2011.

[33] Akira. Onodera and Masaki Takes. Electronic Ferroelectricity in II-VI Semiconductor ZnO. In Advances in Ferroelectrics, volume Chapter 11, pages 231-255. InTech, nov 2012.

[34] L.V. Podrezova, V. Cauda, S. Stassi, G. Cicero, Kh. a. Abdullin, and B. E. Alpysbaeva. Properties of $\mathrm{ZnO}$ nanorods grown by hydrothermal synthesis on conductive layers. Crystal Research and Technology, 49(8):599-605, aug 2014.

[35] David A Scrymgeour and Julia WP Hsu. Correlated piezoelectric and electrical properties in individual $\mathrm{ZnO}$ nanorods. Nano letters, 8(8):2204-2209, 2008.

[36] Ye Sun, D. Jason Riley, and Michael N R Ashfbld. Mechanism of $\mathrm{ZnO}$ nanotube growth by hydrothermal methods on $\mathrm{ZnO}$ film-coated Si substrates. Journal of Physical Chemistry B, 110(31):15186-15192, 2006 .

[37] Stefano Stassi, Valentina Cauda, Carminna Ottone, Angelica Chiodoni, Candido Fabrizio Pirri, and Giancarlo Canavese. Flexible piezoelectric energy nanogenerator based on $\mathrm{ZnO}$ nanotubes hosted in a polycarbonate membrane. Nano Energy, 13:474-481, apr 2015.

[38] Youfan Hu, Long Lin, Yan Zhang, and Zhong Lin Wang. Replacing a Battery by a Nanogenerator with $20 \mathrm{~V}$ Output. Advanced Materials, 24(1):110-114, jan 2012.

[39] Husnu Emrah Unalan, Pritesh Hiralal, Nalin Rupesinghe, Sharvari Dalal, William I Milne, and Gehan a J Amaratunga. Rapid synthesis 
of aligned zinc oxide nanowires. Nanotechnology, 19(25):255608, jun 2008.

[40] Xudong Wang, Jun Zhou, Jinhui Song, Jin Liu, Ningsheng Xu, and Zhong L. Wang. Piezoelectric field effect transistor and nanoforce sensor based on a single ZnO nanowire. Nano Letters, 6(12):2768-2772, 2006.

[41] Marcel Lucas, Wenjie Mai, Rusen Yang, Zhong Lin Wang, and Elisa Riedo. Aspect Ratio Dependence of the Elastic Properties of $\mathrm{ZnO}$ Nanobelts. Nano Letters, 7(5):1314-1317, 2007.

[42] Lukas Schmidt-Mende and Judith L. MacManus-Driscoll. ZnO - nanostructures, defects, and devices. Materials Today, 10(5):40-48, 2007.

[43] Sheng Xu, Nagesh Adiga, Shan Ba, Tirthankar Dasgupta, C. F. Jeff $\mathrm{Wu}$, and Zhong Lin Wang. Optimizing and Improving the Growth Quality of ZnO Nanowire Arrays Guided by Statistical Design of Experiments. ACS Nano, 3(7):1803-1812, jul 2009.

[44] Hongjun Zhou and Stanislaus S. Wong. A facile and mild synthesis of 1-D $\mathrm{ZnO}, \mathrm{CuO}$, and a-Fe2O3 nanostructures and nanostructured arrays. ACS Nano, 2(5):944-958, may 2008.

[45] Carminna Ottone, Katarzyna Bejtka, Angelica Chiodoni, Vivian Farías, Ignazio Roppolo, Giancarlo Canavese, Stefano Stassi, and Valentina Cauda. Comprehensive study of the templating effect on the $\mathrm{ZnO}$ nanostructure formation within porous hard membranes. New Journal of Chemistry, 38(5):2058, 2014.

[46] Satinder K. Sharma, Amritha Rammohan, and Ashutosh Sharma. Templated one step electrodeposition of high aspect ratio n-type $\mathrm{ZnO}$ nanowire arrays. Journal of Colloid and Interface Science, 344(1):1-9, 2010 .

[47] Alina Pruna, Daniele Pullini, and David Busquets Mataix. Influence of Deposition Potential on Structure of ZnO Nanowires Synthesized in Track-Etched Membranes. Journal of The Electrochemical Society, 159(4):E92, 2012. 
[48] Yangyang Zhang, Manoj K. Ram, Elias K. Stefanakos, and D. Yogi Goswami. Synthesis, Characterization, and Applications of $\mathrm{ZnO}$ Nanowires. Journal of Nanomaterials, 2012:1-22, 2012.

[49] Jing-Hua Tian, Jie Hu, Si-Si Li, Fan Zhang, Jun Liu, Jian Shi, Xin Li, Zhong-Qun Tian, and Yong Chen. Improved seedless hydrothermal synthesis of dense and ultralong $\mathrm{ZnO}$ nanowires. Nanotechnology, 22(24):245601, jun 2011.

[50] Bin Liu and Hua Chun Zeng. Hydrothermal Synthesis of ZnO Nanorods in the Diameter Regime of $50 \mathrm{~nm}$. Journal of the American Chemical Society, 125(15):4430-4431, apr 2003.

[51] Bin Cheng and Edward T Samulski. Hydrothermal synthesis of onedimensional $\mathrm{ZnO}$ nanostructures with different aspect ratios. Chemical Communications, (8):986, 2004.

[52] Liang-Yih Chen, Yu-Tung Yin, Ching-hsiang Chen, and Jau-Wern Chiou. Influence of Polyethyleneimine and Ammonium on the Growth of $\mathrm{ZnO}$ Nanowires by Hydrothermal Method. The Journal of Physical Chemistry C, 115(43):20913-20919, nov 2011.

[53] H. L. Cao, X. F. Qian, Q Gong, W. M. Du, X. D. Ma, and Z. K. Zhu. Shape and size controlled synthesis of nanometre $\mathrm{ZnO}$ from a simple solution route at room temperature. Nanotechnology, 17(15):3632-3636, aug 2006.

[54] Nimra Jalali, Joe Briscoe, Yan Zhi Tan, Peter Woolliams, Mark Stewart, Paul M. Weaver, Markys G. Cain, and Steve Dunn. ZnO nanorod surface modification with PDDA/PSS Bi-layer assembly for performance improvement of $\mathrm{ZnO}$ piezoelectric energy harvesting devices. Journal of Sol-Gel Science and Technology, 73(3):544-549, mar 2015.

[55] Nimra Jalali, Peter Woolliams, Mark Stewart, Paul M. Weaver, Markys G. Cain, Steve Dunn, and Joe Briscoe. Improved performance of pn junction-based $\mathrm{ZnO}$ nanogenerators through CuSCN-passivation of ZnO nanorods. Journal of Materials Chemistry A, 2(28):10945, 2014.

[56] Horacio D. Espinosa, Rodrigo A. Bernal, and Majid Minary-Jolandan. A Review of Mechanical and Electromechanical Properties of Piezoelectric Nanowires. Advanced Materials, 24(34):4656-4675, sep 2012. 
[57] Francesca L. Boughey, Timothy Davies, Anuja Datta, Richard A Whiter, Suman-Lata Sahonta, and Sohini Kar-Narayan. Vertically aligned zinc oxide nanowires electrodeposited within porous polycarbonate templates for vibrational energy harvesting. Nanotechnology, 27(28):28LT02, jul 2016.

[58] Xi Chen, Shiyou Xu, Nan Yao, and Yong Shi. 1.6 V nanogenerator for mechanical energy harvesting using PZT nanofibers. Nano Letters, 10(6):2133-2137, 2010.

[59] Canlin Ou, Pedro Enrique Sanchez-Jimenez, Anuja Datta, Francesca L. Boughey, Richard A. Whiter, Suman-Lata Sahonta, and Sohini KarNarayan. Template-Assisted Hydrothermal Growth of Aligned Zinc Oxide Nanowires for Piezoelectric Energy Harvesting Applications. ACS Applied Materials \& Interfaces, 8(22):13678-13683, jun 2016.

[60] A Gruverman, Sergei V Kalinin, et al. Piezoresponse force microscopy and recent advances in nanoscale studies of ferroelectrics. Journal of Materials Science, 41(1):107-116, 2006.

[61] AL Kholkin, Ch Wütchrich, DV Taylor, and N Setter. Interferometric measurements of electric field-induced displacements in piezoelectric thin films. Review of scientific instruments, 67(5):1935-1941, 1996.

[62] Yonatan Calahorra, Michael Smith, Anuja Datta, Hadas Benisty, and Sohini Kar-Narayan. Mapping piezoelectric response in nanomaterials using a dedicated non-destructive scanning probe technique. Nanoscale, 9(48):19290-19297, 2017.

[63] Hong Jin Fan, Woo Lee, Robert Hauschild, Marin Alexe, Gwenaël Le Rhun, Roland Scholz, Armin Dadgar, Kornelius Nielsch, Heinz Kalt, Alois Krost, et al. Template-assisted large-scale ordered arrays of Zno pillars for optical and piezoelectric applications. Small, 2(4):561-568, 2006.

[64] Dimitrios Tamvakos, Serban Lepadatu, Vlad-Andrei Antohe, Athanasios Tamvakos, Paul M Weaver, Luc Piraux, Markys G Cain, and Daniele Pullini. Piezoelectric properties of template-free electrochemically grown ZnO nanorod arrays. Applied Surface Science, 356:12141220, 2015. 
[65] Nikhilendu Tiwary, Ritam Sarkar, V Ramgopal Rao, and Apurba Laha. Piezoresponse force microscopy (PFM) characterization of GaN nanowires grown by Plasma assisted Molecular beam epitaxy (PA-MBE). In Applications of Ferroelectrics, European Conference on Application of Polar Dielectrics, and Piezoelectric Force Microscopy Workshop (ISAF/ECAPD/PFM), 2016 Joint IEEE International Symposium on the, pages 1-4. IEEE, 2016.

[66] Majid Minary-Jolandan, Rodrigo A Bernal, Irma Kuljanishvili, Victor Parpoil, and Horacio D Espinosa. Individual GaN nanowires exhibit strong piezoelectricity in 3D. Nano letters, 12(2):970-976, 2012.

[67] Arseny Kalinin, Valentin Atepalikhin, Oleg Pakhomov, Andrei L Kholkin, and Alexander Tselev. An atomic force microscopy mode for nondestructive electromechanical studies and its application to diphenylalanine peptide nanotubes. Ultramicroscopy, 185:49-54, 2018.

[68] Pu Xian Gao, Jinhui Song, Jin Liu, and Zhong Lin Wang. Nanowire piezoelectric nanogenerators on plastic substrates as flexible power sources for nanodevices. Advanced Materials, 19(1):67-72, 2007.

[69] Ming-Pei Lu, Jinhui Song, Ming-Yen Lu, Min-Teng Chen, Yifan Gao, Lih-Juann Chen, and Zhong Lin Wang. Piezoelectric nanogenerator using p-type ZnO nanowire arrays. Nano letters, 9(3):1223-1227, 2009.

[70] Mohammed Riaz, Jinhui Song, Omer Nur, Zhong Lin Wang, and Magnus Willander. Study of the piezoelectric power generation of $\mathrm{ZnO}$ nanowire arrays grown by different methods. Advanced functional materials, 21(4):628-633, 2011.

[71] Noelle Gogneau, Pascal Chrétien, Elisabeth Galopin, Stephane Guilet, Laurent Travers, Jean-Christophe Harmand, and Frédéric Houzé. GaN nanowires for piezoelectric generators. physica status solidi (RRL)Rapid Research Letters, 8(5):414-419, 2014.

[72] Noelle Gogneau, Pascal Chrétien, Elisabeth Galopin, Stephane Guilet, Laurent Travers, Jean-Christophe Harmand, and Frédéric Houzé. Impact of the GaN nanowire polarity on energy harvesting. Applied Physics Letters, 104(21):213105, 2014. 
[73] N Jamond, P Chrétien, F Houzé, Lu Lu, L Largeau, O Maugain, L Travers, JC Harmand, F Glas, E Lefeuvre, et al. Piezogenerator integrating a vertical array of $\mathrm{GaN}$ nanowires. Nanotechnology, 27(32):325403, 2016.

[74] Chao-Hung Wang, Wei-Shun Liao, Nai-Jen Ku, Yi-Chang Li, YenChih Chen, Li-Wei Tu, and Chuan-Pu Liu. Effects of free carriers on piezoelectric nanogenerators and piezotronic devices made of GaN nanowire arrays. Small, 10(22):4718-4725, 2014.

[75] Min-Yeol Choi, Dukhyun Choi, Mi-Jin Jin, Insoo Kim, Sang-Hyeob Kim, Jae-Young Choi, Sang Yoon Lee, Jong Min Kim, and SangWoo Kim. Mechanically powered transparent flexible charge-generating nanodevices with piezoelectric $\mathrm{ZnO}$ nanorods. Advanced Materials, 21(21):2185-2189, 2009.

[76] Sheng Xu, Yong Qin, Chen Xu, Yaguang Wei, Rusen Yang, and Zhong Lin Wang. Self-powered nanowire devices. Nature nanotechnology, 5(5):366-373, 2010.

[77] Dukhyun Choi, Min-Yeol Choi, Won Mook Choi, Hyeon-Jin Shin, Hyun-Kyu Park, Ju-Seok Seo, Jongbong Park, Seon-Mi Yoon, Seung Jin Chae, Young Hee Lee, et al. Fully rollable transparent nanogenerators based on graphene electrodes. Advanced Materials, 22(19):2187-2192, 2010.

[78] Guang Zhu, Rusen Yang, Sihong Wang, and Zhong Lin Wang. Flexible high-output nanogenerator based on lateral $\mathrm{ZnO}$ nanowire array. Nano letters, 10(8):3151-3155, 2010.

[79] Soon-Shin Kwon, Woong-Ki Hong, Gunho Jo, Jongsun Maeng, TaeWook Kim, Sunghoon Song, and Takhee Lee. Piezoelectric Effect on the Electronic Transport Characteristics of $\mathrm{ZnO}$ Nanowire FieldEffect Transistors on Bent Flexible Substrates. Advanced Materials, 20(23):4557-4562, 2008.

[80] Peng Fei, Ping-Hung Yeh, Jun Zhou, Sheng Xu, Yifan Gao, Jinhui Song, Yudong Gu, Yanyi Huang, and Zhong Lin Wang. Piezoelectric potential gated field-effect transistor based on a free-standing $\mathrm{ZnO}$ wire. Nano letters, 9(10):3435-3439, 2009. 
[81] Wenzhuo Wu, Yaguang Wei, and Zhong Lin Wang. Strain-gated piezotronic logic nanodevices. Advanced Materials, 22(42):4711-4715, 2010.

[82] Zhong Lin Wang. Zinc oxide nanostructures: growth, properties and applications. Journal of Physics: Condensed Matter, 16(25):R829, 2004.

[83] Yamin Leprince-Wang. ZnO Nanostructure Synthesis. Piezoelectric ZnO Nanostructure for Energy Harvesting, pages 21-47, 2015.

[84] Pu Xian Gao, Yong Ding, Wenjie Mai, William L Hughes, Changshi Lao, and Zhong Lin Wang. Conversion of zinc oxide nanobelts into superlattice-structured nanohelices. Science, 309(5741):1700-1704, 2005.

[85] Zhong Lin Wang, Xiang Yang Kong, Yong Ding, Puxian Gao, William L Hughes, Rusen Yang, and Yue Zhang. Semiconducting and piezoelectric oxide nanostructures induced by polar surfaces. Advanced Functional Materials, 14(10):943-956, 2004.

[86] Xiang Yang Kong and Zhong Lin Wang. Spontaneous polarizationinduced nanohelixes, nanosprings, and nanorings of piezoelectric nanobelts. Nano Letters, 3(12):1625-1631, 2003.

[87] Yi-Feng Lin, Jinhui Song, Yong Ding, Shih-Yuan Lu, and Zhong Lin Wang. Piezoelectric nanogenerator using CdS nanowires. Applied Physics Letters, 92(2):022105, 2008.

[88] Lin Dong, Simiao Niu, Caofeng Pan, Ruomeng Yu, Yan Zhang, and Zhong Lin Wang. Piezo-phototronic effect of cdse nanowires. Advanced Materials, 24(40):5470-5475, 2012.

[89] Satish C Rai, Kai Wang, Yong Ding, Jason K Marmon, Manish Bhatt, Yong Zhang, Weilie Zhou, and Zhong Lin Wang. Piezo-phototronic effect enhanced UV/visible photodetector based on fully wide band gap type-II $\mathrm{ZnO} / \mathrm{ZnS}$ core/shell nanowire array. ACS nano, 9(6):64196427, 2015. 
[90] G Arlt and P Quadflieg. Piezoelectricity in iii-v compounds with a phenomenological analysis of the piezoelectric effect. physica status solidi (b), 25(1):323-330, 1968.

[91] Mitiko Miura, Huromu Murata, Yuji Shiro, and Kazuaki Iishi. Ionicity scale and piezoelectricity of crystals with zincblende-and wurtzite-type structure. Journal of Physics and Chemistry of Solids, 42(10):931-936, 1981.

[92] Zhong Lin Wang and Jinhui Song. Piezoelectric nanogenerators based on zinc oxide nanowire arrays. Science (New York, N.Y.), 312(5771):242-6, apr 2006.

[93] X Q Zhang and Et al. Resonant exciton second-harmonic generation in self-assembled $\mathrm{ZnO}$ microcrystallite thin films. Journal of Physics: Condensed Matter, 15(30):5191, 2003.

[94] Zhong Lin Wang and Wenzhuo Wu. Nanotechnology-Enabled Energy Harvesting for Self-Powered Micro-/Nanosystems. Angewandte Chemie International Edition, 51(47):11700-11721, nov 2012.

[95] Zhou Li, Rusen Yang, Min Yu, Fan Bai, Cheng Li, and Zhong Lin Wang. Cellular level biocompatibility and biosafety of $\mathrm{ZnO}$ nanowires. Journal of Physical Chemistry C, 112(51):20114-20117, 2008.

[96] Joe Briscoe, Mark Stewart, Melvin Vopson, Markys Cain, Paul M. Weaver, and Steve Dunn. Nanostructured p-n junctions for kinetic-toelectrical energy conversion. Advanced Energy Materials, 2(10):12611268, 2012.

[97] Lori E. Greene, Matt Law, Joshua Goldberger, Franklin Kim, Justin C. Johnson, Yanfeng Zhang, Richard J. Saykally, and Peidong Yang. LowTemperature Wafer-Scale Production of $\mathrm{ZnO}$ Nanowire Arrays. Angewandte Chemie International Edition, 42(26):3031-3034, 2003.

[98] Zhiyuan Gao, Yong Ding, Shisheng Lin, Yue Hao, and Zhong Lin Wang. Dynamic fatigue studies of $\mathrm{ZnO}$ nanowires by in-situ transmission electron microscopy. Physica Status Solidi - Rapid Research Letters, 3(78):260-262, 2009. 
[99] Vivian Farías Rivera, Florian Auras, Paolo Motto, Stefano Stassi, Giancarlo Canavese, Edvige Celasco, Thomas Bein, Barbara Onida, and Valentina Cauda. Length-Dependent Charge Generation from Vertical Arrays of High-Aspect-Ratio ZnO Nanowires. Chemistry - A European Journal, 19(43):14665-14674, oct 2013.

[100] Guang Zhu, Aurelia C. Wang, Ying Liu, Yusheng Zhou, and Zhong Lin Wang. Functional electrical stimulation by nanogenerator with $58 \mathrm{v}$ output voltage. Nano Letters, 12(6):3086-3090, 2012.

[101] Zhitao Han, Sisi Li, Jinkui Chu, and Yong Chen. Controlled growth of well-aligned $\mathrm{ZnO}$ nanowire arrays using the improved hydrothermal method. Journal of Semiconductors, 34(6):063002, 2013.

[102] Valentina Cauda, Stefano Stassi, Andrea Lamberti, Marco Morello, Candido Fabrizio Pirri, and Giancarlo Canavese. Leveraging ZnO morphologies in piezoelectric composites for mechanical energy harvesting. Nano Energy, 18:212-221, 2015.

[103] Joe Briscoe and Steve Dunn. Piezoelectric nanogenerators a review of nanostructured piezoelectric energy harvesters. Nano Energy, 14:1529, may 2015.

[104] Joe Briscoe, Nimra Jalali, Peter Woolliams, Mark Stewart, Paul M Weaver, Markys Cain, and Steve Dunn. Measurement techniques for piezoelectric nanogenerators. Energy $\&$ Environmental Science, 6(10):3035, 2013.

[105] E.S. Nour, M.O. Sandberg, M. Willander, and O. Nur. Handwriting enabled harvested piezoelectric power using $\mathrm{ZnO}$ nanowires/polymer composite on paper substrate. Nano Energy, 9:221-228, 2014.

[106] Sung-ho Shin, Young-hwan Kim, Min Hyung Lee, Joo-yun Jung, Jae Hun Seol, and Junghyo Nah. Lithium-Doped Zinc Oxide NanowiresPolymer Composite for High Performance Flexible Piezoelectric Nanogenerator. ACS Nano, 8(10):10844-10850, oct 2014.

[107] Youfan Hu, Yan Zhang, Chen Xu, Guang Zhu, and Zhong Lin Wang. High-Output Nanogenerator by Rational Unipolar Assembly of Conical Nanowires and Its Application for Driving a Small Liquid Crystal Display. Nano Letters, 10(12):5025-5031, dec 2010. 
[108] Minbaek Lee, Chih-Yen Chen, Sihong Wang, Seung Nam Cha, Yong Jun Park, Jong Min Kim, Li-Jen Chou, and Zhong Lin Wang. A Hybrid Piezoelectric Structure for Wearable Nanogenerators. Advanced Materials, 24(13):1759-1764, apr 2012.

[109] Yi-Feng Lin, Jinhui Song, Yong Ding, Shih-Yuan Lu, and Zhong Lin Wang. Alternating the output of a CdS nanowire nanogenerator by a white-light-stimulated optoelectronic effect. Advanced Materials, 20(16):3127-3130, 2008.

[110] Guozhang Dai, Haiyang Zou, Xingfu Wang, Yuankai Zhou, Peihong Wang, Yong Ding, Yan Zhang, Junliang Yang, and Zhong Lin Wang. Piezo-phototronic Effect Enhanced Responsivity of Photon Sensor Based on Composition-Tunable Ternary CdS x Se1-x Nanowires. ACS Photonics, 4(10):2495-2503, 2017.

[111] Te-Chien Hou, Ya Yang, Zong-Hong Lin, Yong Ding, Chan Park, Ken C Pradel, Lih-Juann Chen, and Zhong Lin Wang. Nanogenerator based on zinc blende CdTe micro/nanowires. Nano Energy, 2(3):387-393, 2013.

[112] IL Guy, S Muensit, and EM Goldys. Extensional piezoelectric coefficients of gallium nitride and aluminum nitride. Applied Physics Letters, 75(26):4133-4135, 1999.

[113] Mingzeng Peng, Zhou Li, Caihong Liu, Qiang Zheng, Xieqing Shi, Ming Song, Yang Zhang, Shiyu Du, Junyi Zhai, and Zhong Lin Wang. Highresolution dynamic pressure sensor array based on piezo-phototronic effect tuned photoluminescence imaging. ACS nano, 9(3):3143-3150, 2015.

[114] Mingzeng Peng, Yudong Liu, Aifang Yu, Yang Zhang, Caihong Liu, Jingyu Liu, Wei Wu, Ke Zhang, Xieqing Shi, Jinzong Kou, et al. Flexible self-powered GaN ultraviolet photoswitch with piezo-phototronic effect enhanced on/off ratio. ACS nano, 10(1):1572-1579, 2015.

[115] Chunhua Du, Chunyan Jiang, Peng Zuo, Xin Huang, Xiong Pu, Zhenfu Zhao, Yongli Zhou, Linxuan Li, Hong Chen, Weiguo Hu, et al. PiezoPhototronic Effect Controlled Dual-Channel Visible light Communication (PVLC) Using InGaN/GaN Multiquantum Well Nanopillars. Small, 11(45):6071-6077, 2015. 
[116] Weidong Song, Xingfu Wang, Chao Xia, Rupeng Wang, Liangliang Zhao, Dexiao Guo, Hang Chen, Jiakai Xiao, Shichen Su, and Shuti Li. Improved photoresponse of a-axis GaN microwire/p-polymer hybrid photosensor by the piezo-phototronic effect. Nano Energy, 33:272-279, 2017.

[117] Sheng-Chieh Tsai, Cheng-Hsueh Lu, and Chuan-Pu Liu. Piezoelectric effect on compensation of the quantum-confined Stark effect in InGaN/GaN multiple quantum wells based green light-emitting diodes. Nano Energy, 28:373-379, 2016.

[118] Chi-Te Huang, Jinhui Song, Wei-Fan Lee, Yong Ding, Zhiyuan Gao, Yue Hao, Lih-Juann Chen, and Zhong Lin Wang. Gan nanowire arrays for high-output nanogenerators. Journal of the American Chemical Society, 132(13):4766-4771, 2010.

[119] Long Lin, Chen-Ho Lai, Youfan Hu, Yan Zhang, Xue Wang, Chen Xu, Robert L Snyder, Lih-J Chen, and Zhong Lin Wang. High output nanogenerator based on assembly of GaN nanowires. Nanotechnology, 22(47):475401, 2011.

[120] Guocheng Liu, Songrui Zhao, Robert DE Henderson, Zoya Leonenko, Eihab Abdel-Rahman, Zetian Mi, and Dayan Ban. Nanogenerators based on vertically aligned InN nanowires. Nanoscale, 8(4):2097-2106, 2016.

[121] E Klier and J Nedbal. Piezoelectric resonators of InP:Fe. Czechoslovak journal of physics, 44(6):575-584, 1994.

[122] J Nedbal and E Klier. Piezoelectric resonators of semiinsulating GaAs. physica status solidi (a), 148(1):329-340, 1995.

[123] Jasprit Singh. Electronic and optoelectronic properties of semiconductor structures. Cambridge University Press, 2007.

[124] Hanan YS Al-Zahrani, Joydeep Pal, Max A Migliorato, Geoffrey Tse, and Dapeng Yu. Piezoelectric field enhancement in III-V core-shell nanowires. Nano Energy, 14:382-391, 2015.

[125] IP Soshnikov, Dm E Afanasev, VA Petrov, GE Cirlin, AD Bouravlev, Yu B Samsonenko, A Khrebtov, EM Tanklevskaya, and IA Seleznev. 
Piezoelectric effect in GaAs nanowires. Semiconductors, 45(8):1082, 2011.

[126] Volodymyr V Lysak, Ilya P Soshnikov, Erkki Lahderanta, and George E Cirlin. Piezoelectric effect in wurtzite GaAs nanowires: Growth, characterization, and electromechanical 3D modeling. physica status solidi (a), 213(11):3014-3019, 2016.

[127] Prokhor A. Alekseev, Vladislav A. Sharov, Pavel Geydt, Mikhail S. Dunaevskiy, Volodymyr V. Lysak, George E. Cirlin, Rodion R. Reznik, Artem I. Khrebtov, Ilya P. Soshnikov, and Erkki Lhderanta. Piezoelectric current generation in wurtzite gaas nanowires. physica status solidi (RRL) Rapid Research Letters, 12(1):1700358-n/a, 2018. 1700358. 